https://doi.org/10.11646/zootaxa.4231.3.1

http://zoobank.org/urn:lsid:zoobank.org:pub:1226613C-0001-4369-93C9-3D0B1A40BE99

\title{
Distribution of haploniscids (Isopoda, Asellota, Haploniscidae) in Icelandic waters, with description of Haploniscus astraphes n. sp. from the Iceland basin and the Southeast Atlantic Ocean
}

\author{
WIEBKE BRÖKELAND ${ }^{1} \&$ JÖRUNDUR SVAVARSSON ${ }^{2,3}$ \\ ${ }^{I}$ Senckenberg am Meer Wilhelmshaven, Abt.DZMB, Südstrand 44, 26382 Wilhelmshaven, Germany.E-mail: w.broekeland@gmx.net \\ ${ }^{2}$ Marine Biology Centre, Institute of Biology, University of Iceland, Sturlugata 7, 101 Reykjavik, Iceland; ${ }^{3}$ The University of Iceland's \\ Research Centre in Suðurnes, Garðvegi 1, 245 Sandgerði, Iceland.E-mail: jorundur@hi.is
}

\begin{abstract}
Ten species of Haploniscidae Hansen, 1916 were sampled in Icelandic waters during expeditions in the framework of the BIOICE project. Nine of these were known from the North Atlantic Ocean, i.e. Haploniscus aduncus Lincoln, 1985, H. ampliatus Lincoln, 1985, Haploniscus angustus Lincoln, 1985, H. bicuspis (Sars, 1877), H. foresti Chardy, 1974, H. hamatus Lincoln, 1985, H. spinifer Hansen, 1916, Antennuloniscus simplex Lincoln, 1985 and Chauliodoniscus armadilloides (Hansen, 1916). All but H. bicuspis and H. angustus were restricted to the Atlantic Ocean south of the GreenlandIceland-Faeroe Ridge (GIF Ridge), while H. bicuspis occurred at considerable depth ranges both north and south of the GIF Ridge. A new species, Haploniscus astraphes n. sp., is described based on material from the Denmark Strait, North Atlantic and the Guinea Basin, South Atlantic. H. astraphes n. sp. belongs to a group of Haploniscus species closely related to the genus Antennuloniscus and shares several characters with species from that genus, especially the spine row on pleopod 1, the stout sensory seta on the carpus of pereopod 7 and characters of the antennae. H. astraphes $\mathbf{n}$. sp. is characterized by a rectangular body shape, the straight frontal margin of the head and the strongly convex posterior margin of the pleotelson.
\end{abstract}

Key words: BIOICE, DIVA, Haploniscidae, Haploniscus, Antennuloniscus, Chauliodoniscus, Greenland-Iceland-Faeroe Ridge

\section{Introduction}

The northernmost part of the North Atlantic Ocean is characterized by the extensive Greenland-Iceland-Faeroe Ridge (GIF Ridge). This is the only large submarine ridge in the North Atlantic Ocean stretching in easterlywesterly direction, separating the deeper parts of the Nordic Seas and the Arctic Ocean from the deep North Atlantic Ocean. This ridge shapes the distribution of water masses in this area, having generally colder water masses (often $<0^{\circ} \mathrm{C}$ ) to the north of the ridge, while warmer water masses (often $>0{ }^{\circ} \mathrm{C}$ ) characterize the area south of the ridge (see Stefánsson 1962; Hansen \& Østerhus 2000). The colder water masses flow southward, either along East Greenland or crossing the ridge usually in deep channels (deepest channel of 840 m; Hansen \& Østerhus 2000), while the warmer water masses flow northwards usually in shallower waters.

The ridge and the associated water masses shape the distribution and diversity of benthic invertebrates in the area (Svavarsson et al. 1993). Most of the species have their distribution either to the north or to the south of the ridge (see data in Hansen 1916; Negoescu \& Svavarsson 1997; Weisshappel 2001; Dijkstra et al. 2009; Brix \& Svavarsson 2010). The ridge may accordingly serve as barrier for dispersal of deep-water species and thus prevent species from entering the Nordic Seas and the Arctic Ocean.

The aim of the international BIOICE project was partly to explore the distribution and species composition in Icelandic waters north and south of the GIF Ridge. The family Haploniscidae is particularly interesting for evaluating patterns of distribution in this area. The family is one of the most pronounced deep-sea families among the asellote isopod crustaceans (see Svavarsson et al. 1993) currently holding 142 species (Boyko et al. 2008 
onwards; Ahyong et al. 2011) of small isopods mostly restricted to the deep-sea. Among the many species sampled during the BIOICE project were ten species of the Haploniscidae. One of the species was new to science while the other nine were already known, i.e. H. aduncus Lincoln, 1985, H. ampliatus Lincoln, 1985, H. angustus Lincoln, 1985, H. bicuspis (Sars, 1877), H. foresti Chardy, 1974, H. hamatus Lincoln, 1985, H. spinifer Hansen, 1916, Antennuloniscus simplex Lincoln, 1985 and Chauliodoniscus armadilloides (Hansen, 1916). Here we describe the new haploniscid species and examine the distribution of the other species in the area.

\section{Material and methods}

The study area. The area is the northernmost part of the North Atlantic Ocean and the southern part of the Nordic Seas (the Iceland, Greenland and Norwegian Seas). This area is characterized by the extensive submarine ridge extending from Greenland to the Faeroe Islands, the Greenland-Iceland-Faeroe Ridge (GIF Ridge; often referred to as the Greenland-Iceland-Scotland Ridge; the GIS Ridge). The deepest saddle depth of the ridge is between the Faeroe Islands and Scotland at around 840 meters, while the deepest saddle depth in the Denmark Strait between Greenland and Iceland is around 620 meters. Between Iceland and the Faeroes the deepest saddle depth is around 480 meters (Hansen \& Østerhus 2000).

The area is characterized by several primary water masses, often overlying each other (see Stefánsson 1962; Hansen \& Østerhus 2000; Malmberg \& Valdimarsson 2003), and all differing in either temperature (total range $=$ -0.9 to $12^{\circ} \mathrm{C}$ ) and salinity (total range $=34.30-35.45$ ). These are modified North Atlantic Waters (MNAW; 7.0 $8.5^{\circ} \mathrm{C}$, salinity $35.10-35.30$ ), Labrador Sea Water (LSW; 3-4 $4^{\circ} \mathrm{C}$, salinity 34.90-34.95), Iceland Sea Overflow Water (ISOW; $2-3^{\circ} \mathrm{C}$, salinity 34.85-35.00), Modified East Icelandic Water (MEIW; 1-3 ${ }^{\circ} \mathrm{C}$, salinity 34.70-34.90), Norwegian Sea Deep Water (NSDW; $<-0.5^{\circ} \mathrm{C}$, salinity $<-34.90$ ), Norwegian Sea Arctic Intermediate Water (NSAIW; $-0.5-0.5^{\circ} \mathrm{C}$, salinity $34.85-34.90$ ), Arctic/Polar Water (A/PW; $0-2^{\circ} \mathrm{C}$, salinity $34.30-34.90$ ) and Coastal Water $\left(\mathrm{CW} ; 5-12^{\circ} \mathrm{C}\right.$, salinity $\left.<34.50\right)$.

Additional material of Haploniscus astraphes n. sp. was sampled during the DIVA 2 expedition (DIVA: Latitudinal Gradients of Deep-Sea BioDIVersity in the Atlantic Ocean) in the Guinea and Angola Basins in the southeast Atlantic Ocean in 2005. The major bottom water mass in these basins is the North Atlantic Deep Water (NADW) which partly mixes with Circumpolar Deep Water (CDW) passing through the Romanche and Chain Fracture Zones in the Mid-Atlantic Ridge.

Sampling, identifying and measuring. The BIOICE samples were taken with a modified Rothlisberg-Pearcy epibenthic sled (RP sled; Rothlisberg \& Pearcy 1977, Brattegard \& Fosså 1991), a Sneli sled (Sneli 1998), a Triangle dredge and an Agassiz trawl. The RP sled samples were elutriated through a $0.5 \mathrm{~mm}$ sieve, and then the remaining sediment and animals were sieved through a series of sieves (4, 2, 1 and $0.5 \mathrm{~mm})$ and preserved in 5\% buffered formalin. The Sneli sled samples were sieved through 2, 1 and $0.5 \mathrm{~mm}$ sieves, while the Agassiz samples and the Triangle dredge samples were hand-picked on deck. Most specimens were collected in the RP sled. The DIVA samples were taken with a Brenke sledge (Brenke 2005) and preserved in ethanol having been sorted upon arrival at the deck.

The specimens were identified, measured and dissected using a Leica MZ APO12.5 or a Leica MZ9.5 dissecting microscope, drawings were prepared using Leica compound microscopes (DMLS2, DM2500) equipped with camera lucida.

Body length was measured in lateral view along the midline from the anterior margin of the head to the posterior margin of the pleotelson. Body segment and limb article lengths were also measured along the midline. Other measurements refer to the greatest length/width of an article or segment. The differentiation in peduncular and flagellar articles of the antennae follows Lincoln (1985a) and Wägele (1983). Allocation to ontogenetic stages follows Brökeland (2010). Examined DIVA specimens are deposited at the Zoological Museum Hamburg (ZMH), while the BIOICE specimens of Haploniscus astraphes and specimens of the other haploniscid species are deposited at the Icelandic Museum of Natural History (IMNH).

\section{Taxonomy}

Family Haploniscidae Hansen, 1916 


\section{Haploniscus astraphes $\mathrm{n} . \mathrm{sp}$.}

(Figs 1-9)

Holotype: DIVA2-HA307, male stage V, $1.01 \mathrm{~mm}$, RV Meteor, M63/2, DIVA 2 expedition, EBS station 89-6, $0^{\circ} 42.95^{\prime} \mathrm{N}, 5^{\circ} 31.29^{\prime} \mathrm{W}, 5142 \mathrm{~m}$, Guinea Basin, southeast Atlantic, ZMH K-42450.

Paratypes: DIVA2-HA308, 1 manca stage III, same locality as holotype, ZMH K-42451; DIVA2-HA515, 1 male stage V, $1.04 \mathrm{~mm}$, DIVA2-HA414, 1 female stage IV or V, $1.06 \mathrm{~mm}$, DIVA2-HA415, 1 manca stage III, 0.92 mm, EBS station 90-7, 040.49’ N, 5²9.71’ W, 5142 m, Guinea Basin, ZMH K-42502; DIVA2-HA153, 1 female stage IV or V, 1.24 mm, EBS station 45-3, 953.76’S, 053.60’'E, 5657 m, Angola Basin, ZMH K-42503.

BIOICE station 2909, RV Bjarni Scemundsson, North Atlantic: 2 females stage IV or V, 1.25 and $1.09 \mathrm{~mm}$, RP sled, $65^{\circ} 15.61^{\prime} \mathrm{N}, 28^{\circ} 50.15^{\prime} \mathrm{W}, 1300 \mathrm{~m}, 25$ August $1996,3.96^{\circ} \mathrm{C}$, Denmark Strait, south of Greenland-Iceland Ridge, IMNH 10370.

Etymology. The Greek astraphes, "straight", refers to the straight outlines of head and pereon in this species.

Diagnosis. Body rectangular. Head rectangular in males, trapezoidal in females. Vertex straight, without rostrum. Clypeus slightly prolonged, dorsally keeled. Pleotelson terminal margin strongly convex. Antenna 2 peduncular article 1 small, article 3 with long tooth, curved backwards, articles 5 and 6 short, not fused, article 6 with strong apical projection, flagellum small with 8-9 articles. Mandibular palp article 3 with 6 pectinate setae. Maxilliped with 2 retinaculae. Outer apical comb on carpus of pereopods 4-6 composed of large spines, much larger than inner comb; dorsal sensory stout seta on carpus of pereopod 7; distalmost ventral scale on propodus drawn out towards dactylus. Pleopod 2 of males with short stylet of the endopod, operculum of females nearly circular. Pleopod 3 exopod with 3 simple setae. Uropods stout, ramus length about 1.8 width.

Description of male (stage V). Body (Fig. 1) length 3.3 width, rectangular, lateral margins nearly parallel. Head length 0.7 width, rectangular; vertex straight, without rostrum; anterolateral margins slant in lateral view; clypeus slightly prolonged, keeled. Pereonites 4 and 5 longest, pereonite 7 shortest; anterolateral angles of pereonites $2-5$ acute. Pleotelson as wide as pereonite 7 basally, length 1.1 width, lateral margins convex, slightly concave distally; terminal margin strongly convex; pleotelson processes short, slightly exceeding terminal margin; dorsal surface with two indistinct keels, ventral surface with faint cuticular ledge around branchial chamber.

Antenna 1 (Fig. 4A) length 0.3 body length, peduncular article 1 broadest, length 1.6 width, with 2 penicillate setae distally; article 2 length 0.9 article 1 length, 2.2 width, with 1 simple and 4 penicillate setae distally; article 3 length 0.4 article 1 length, width 0.4 article 1 width, with 1 simple seta distally. Flagellum with 4 articles; article 1 shortest, with 2 penicillate setae distally; article 2 length 2.0 article 1 length, with 1 simple seta, article 3 length 1.45 article 1 length, with 1 aesthetasc and 1 simple seta, article 4 length 2.3 article 1 length, with 2 aesthetascs, 1 penicillate seta and 3 simple setae.

Antenna 2 (Fig. 4B) length 0.6 body length, peduncular article 1 minute (lost during dissection), article 2 longer, with 1 simple seta; article 3 length 1.7 article 2 length, 1.9 width, with 3 simple setae, dorsal tooth slightly longer than article width, curved backwards, with at least one simple seta; article 4 length 0.8 article 2 length, as wide as long, with 1 simple seta; article 5 short, length 1.1 article 2 length, 1.4 width, with about 4 simple setae in distal half; article 6 short, length without tooth 1.1 article 2 length, 1.65 width, with at least 3 penicillate setae and about 11 simple setae, distal tooth length about 0.7 article length. Flagellum small, total length 2.6 peduncular article 6 length, with 9 articles, decreasing in length and width from articles 1-7, articles 8 and 9 slender; with up to 5 simple setae per article.

Mandible (Figs 5A, B) incisor with 5 cusps; lacinia mobilis of left mandible with 5 teeth; spine row with 2 serrated and 2 setulated spines, spine row of right mandible with 3 serrated and 3 setulated spines. Molar with tooth row and opposing cuticular ledge indistinctly separated into teeth, 4-5 strong setae proximally of tooth row, accessory teeth row proximally of cuticular ledge and 2-3 setae on molar trunk. Palp slightly shorter than mandibular body, article 1 with 1 simple distal seta, article 2 with 3 pectinate setae distally, article 3 with 6 pectinate setae along inner margin.

Maxilla 1 (Fig. 6A) lateral lobe length 3.5 width, distal margin with 12 stout setae, outer margin with rows of slender setae, inner margin with 2 setae distally, surface with few scattered rows of small setae; mesial lobe length about 0.7 outer lobe length, width 0.6 outer lobe width, distal margin with 2 stout 4 long and several smaller setae, surface and outer margin with several slender setae. 

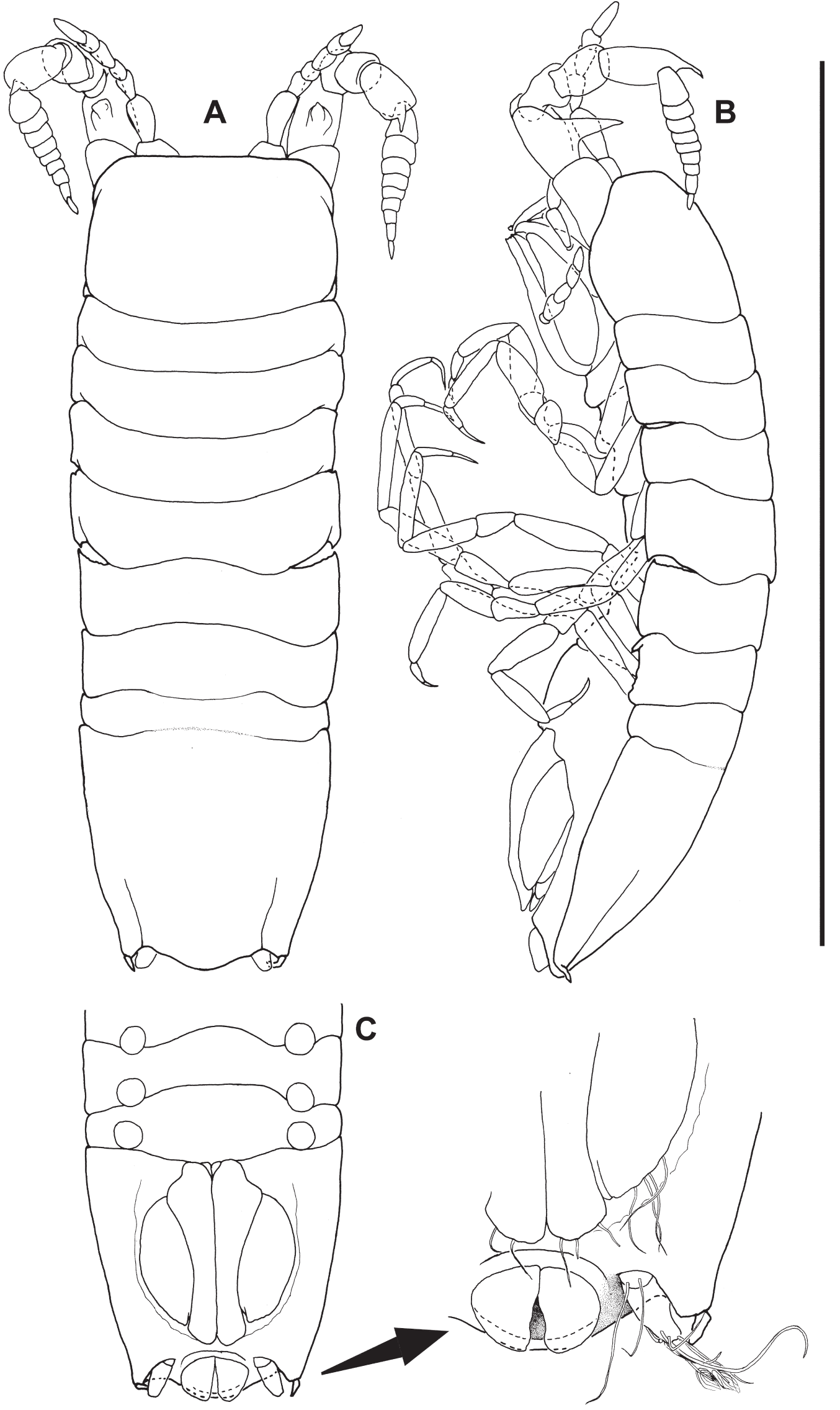

FIGURE 1. Haploniscus astraphes n. sp. male holotype, ZMH K-42450. A, habitus, dorsal view; B, habitus, lateral view; C, posterior body, ventral view. Scale bar $=1 \mathrm{~mm}$. 

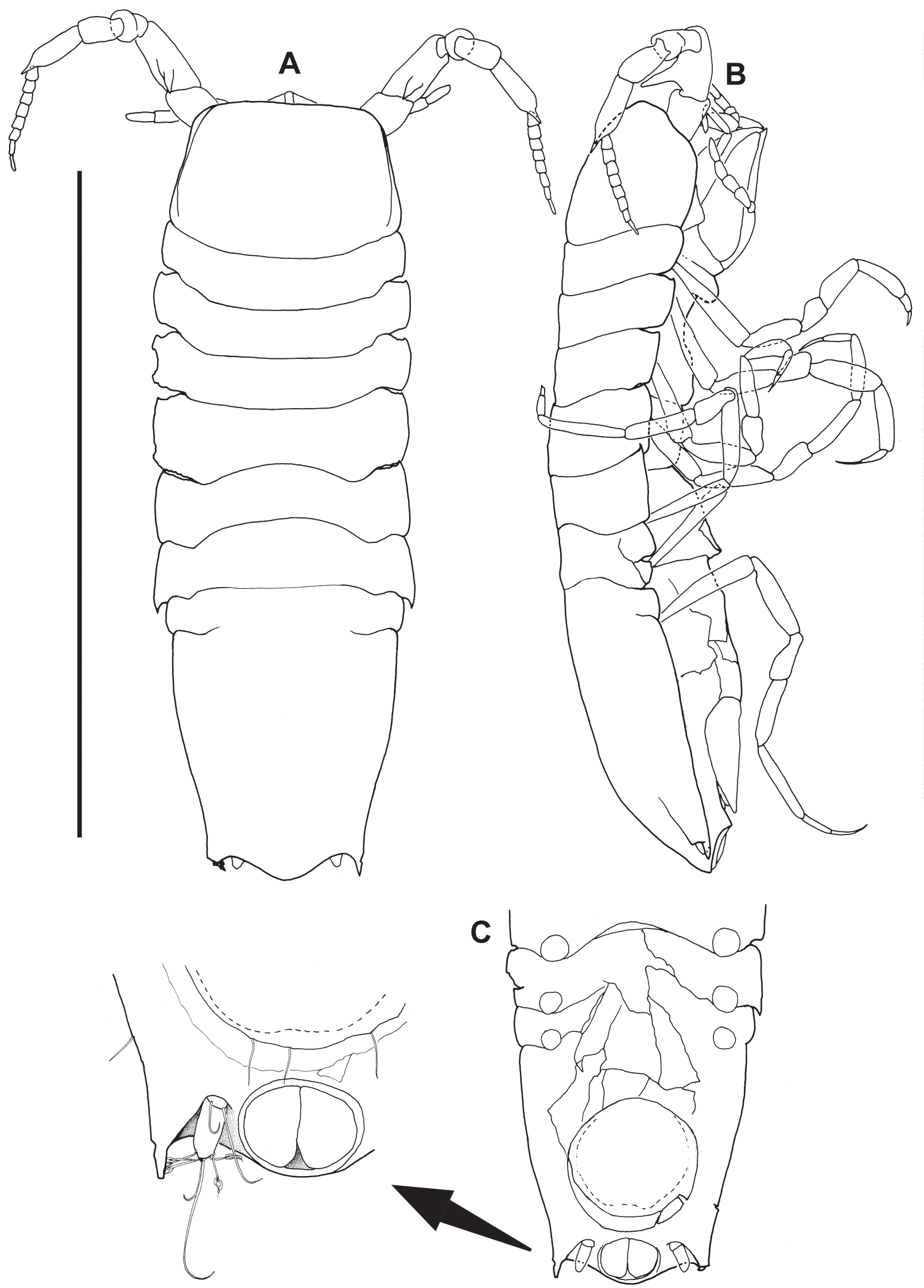

FIGURE 2. Haploniscus astraphes n. sp. female paratype, ZMH K-42503. A, habitus, dorsal view; B, habitus, lateral view; C, posterior body, ventral view. Scale bar $=1 \mathrm{~mm}$. 

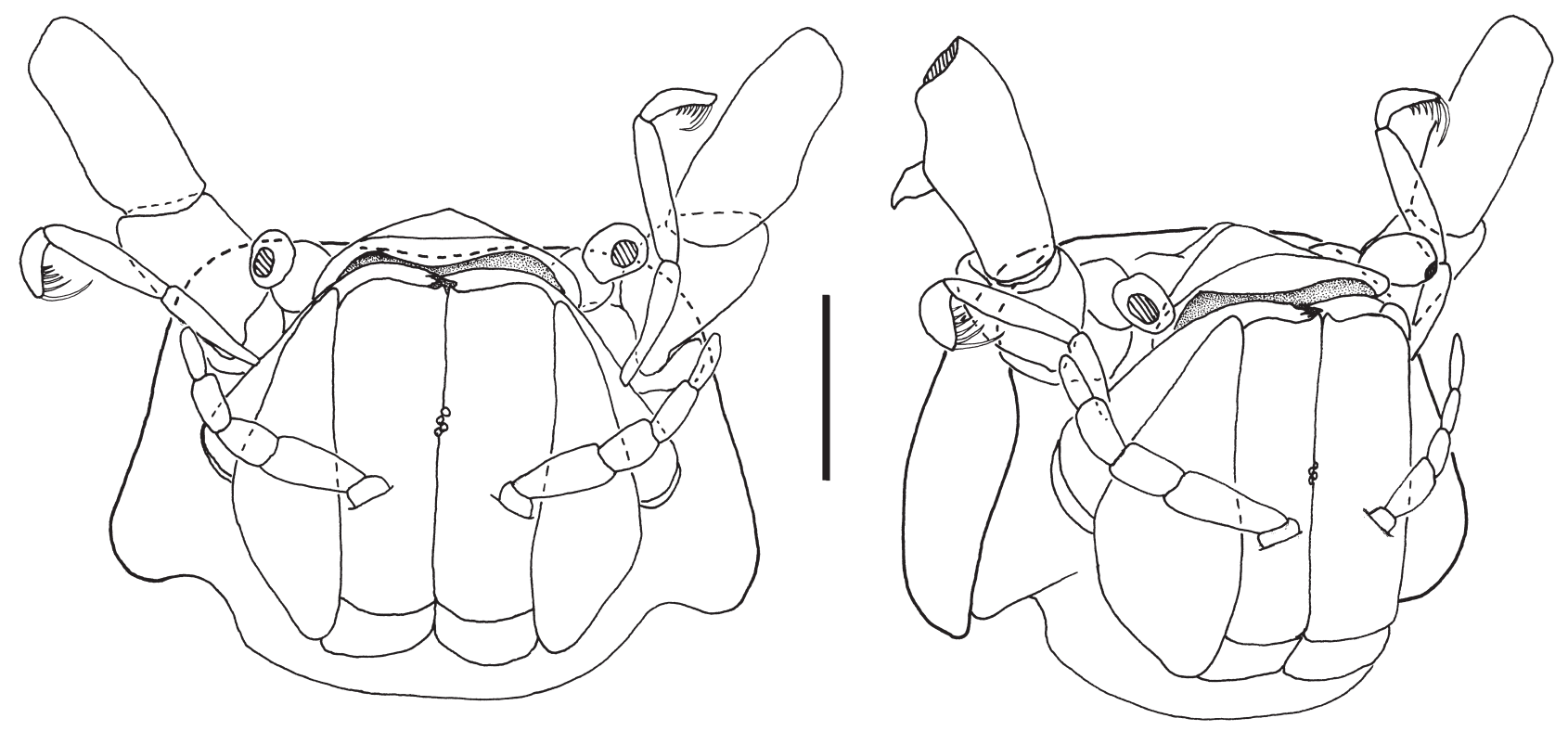

FIGURE 3. Haploniscus astraphes n. sp. female paratype, ZMH K-42503. Head, A, ventral view; B, lateroventral view. Scale bar $=0.1 \mathrm{~mm}$.

Maxilla 2 (Fig. 6B) lateral and middle lobes subequal in length and width, length about 5.5 width; mesial lobe width 1.8 middle lobe width, length 1.3 middle lobe length, 3.9 width. Proximal inner margin of mesial lobe with about 3 long setae; surface and distal inner margin with rows of stout and slender setae; distal margin with 2 short serrated stout setae and 5 strong setae. Lateral and middle lobe each with 1 small stout seta and 2 long and 1 shorter serrated stout setae distally. Inner margin of middle endite with several strong setae, outer margin of lateral endite with numerous slender setae.

Pereopods (Figs 7, 8): Basis with 1-2 long simple setae dorsally and 1 simple seta ventrally (probably broken off on pereopod 7); basis of pereopods 2-7 with 1 penicillate seta. Ischium of pereopods 1-4 with 1 seta, ischium of pereopods 5-7 with 2 setae ventrally. Merus with 2 setae distoventrally and distodorsally each, merus of pereopod 6 with 1 seta on ventral margin. Ventral margins of carpus, propodus and dactylus fringed by comb-like scale rows, distalmost scale on propodus drawn out towards dactylus; surface of carpus and propodus with some scale rows. Carpus of pereopod 1 with 3 setae ventrally, carpus of pereopods 2-7 with 2 setae ventrally; distal carpus with 1 simple seta dorsally, distal carpus of pereopods 2-7 with 1 penicillate seta (probably broken off on pereopod 3) dorsally, carpus of pereopod 7 with one very stout sensory seta; apical combs of peropods 2-7 of different size, outer comb distinctly larger, more spinose than inner comb, outer combs of pereopods 4-6 composed of very large spines. Propodus with 2-4 setae ventrally, pereopods 1-3 with 1 long simple seta distodorsally, seta shorter on remaining pereopods (broken off on pereopod 4), pereopods $2-7$ with penicillate seta distodorsally (broken off on pereopods 4 and 6); with small apical comb. Dactylus with 2-5 setae near insertion of unguis and triangular accessory claw.

Pleopod 1 (Fig. 9A) length 0.8 ventral pleotelson length, 1.85 width, neck (narrowest part) width 0.5 maximum width. Lateral margins concave, distal margins rounded, smooth, with some setae.

Pleopod 2 (Fig. 9B) basipod semicircular, length 1.8 width, distal two thirds of lateral margin with long setae, endopod short, not reaching tip of basipod.

Uropod (Fig. 1C) stout, ramus length 1.8 width, reaching terminal margin of pleotelson.

Description of female (stage IV or V). Body (Figs 2, 3) length 3.2 width. Head trapezoidal, tapering towards vertex; pereonite 7 and pleotelson slightly narrower than pereonite 6 . Pleotelson length 1.3 width, lateral margins convex basally, concave distally; terminal margin strongly convex; pleotelson processes short, reaching terminal margin; dorsal surface with two indistinct keels.

Antenna 1 (Fig. 4D) peduncular article 1 with 3-4 penicillate setae distally. Flagellum with 3 articles; article 1 shortest, with 2 penicillate setae distally; articles 2 and 3 of subequal length, each 2.0 article 1 length, article 2 with 1 simple seta, article 3 with 2 aesthetascs, 1 penicillate seta and 1 simple seta. 
Antenna 2 (Fig. 4C) peduncular article 1 minute, article 2 with 2 simple setae; article 3 length 1.55 article 2 length, 2.25 width, with 1 simple setae, dorsal tooth slightly longer than article width, with at least one simple seta; article 4 length 1.3 width; article 5 length 0.8 article 2 length, 1.2 width, with about 2 simple setae distally; article 6 , without tooth as long as article 2, length 2.1 width, with at least 2 penicillate setae and 7 simple setae. Flagellum total length 2.3 peduncular article 6 length, with 8 articles, articles 2-8 of subequal length, decreasing in width, more slender than in male; with up to 4 simple setae per article.

Maxilliped (Fig. 6C) endite apical margin with at least 2 small fan setae, several small stout setae and simple setae; apical medial margin with 1 serrated and 1 simple stout seta dorsally and a row of strong setae on ventral surface; few simple setae on dorsal surface; 2 retinaculae. Epipod slightly longer than endite.

Pleopod 2 (Fig. 9C) nearly circular, shoulders indistinct, slightly wider than long, ventral suface smooth with shallow depression basally, lateral margins with 1 seta each, distal margin with 6 setae.

Pleopod 3 (Fig. 9D) endopod length 1.65 width. Exopod length 0.4 endopod length, 1.2 width, with 3 simple setae.

Pleopod 4 (Fig. 9E) endopod length 1.4 width. Exopod length 0.45 endopod length, 3.1 width, plumose seta length about 2.5 exopod length.

Pleopod 5 (Fig. 9F) length 2.1 width.

Remarks. Only a few specimens of Haploniscus astraphes $\mathbf{n}$. sp. were found in both localities, the Guinea and Angola Basins and the North Atlantic (Fig. 10A, dot), and the two specimens from the Denmark Strait were both juvenile females. With such a long distance and a depth difference of about $3800 \mathrm{~m}$ between the localities it might seem doubtful that the specimens from the North Atlantic Ocean actually belong to the same species as those from the South Atlantic Ocean. Examination of the available specimens revealed no morphological differences, as to justify the erection of a separate species. Therefore the specimens from the North Atlantic are allocated to $H$. astraphes $\mathbf{n}$. sp. until more material allows the analysis of further characters.

H. astraphes n. sp. strongly resembles species of the genus Antennuloniscus Menzies, 1962. The latter genus has been characterized by the following apomorphies: clypeus strongly prolonged, article 1 of antenna 2 small, articles 5 and 6 of antenna 2 fused (suture may be visible), and pleopod 1 with spine row near distal end of transverse groove (Brökeland 2006).

H. astraphes n. sp. is similar in the shape of the head and body to Antennuloniscus simplex Lincoln, 1985, which also occurs in Icelandic waters. This latter species is different from other Antennuloniscus species in having the clypeus less prolonged and the antenna 2 articles 5 and 6 , while definitely fused, are separated by a more distinct suture than in most other species of the genus. The flagellum of antenna 2 is, however, smaller in $A$. simplex than in $H$. astraphes and has only six articles, while the flagellum of $H$. astraphes has eight or nine articles.

At least three other species of Haploniscus, i.e. H. rostratus (Menzies, 1962), H. ingolfi Wolff, 1962, and H. pygmaeus Birstein, 1969, seem to be closely related to Antennuloniscus (Brökeland 2010). Together with $H$. astraphes $\mathbf{n}$. sp. they share some characters with the Antennuloniscus species, such as article 3 of antenna 2 being longer than wide, article 6 of antenna 2 having a large terminal projection and therefore the flagellum being inserted subapically. An interesting feature is the location of the dorsal sensory stout seta on the carpus of pereopod 7 instead of pereopod 6, where it is located in the majority of haploniscid species. As far as could be determined from the species descriptions, Antennuloniscus species also have the sensory stout seta on pereopod 7, as seen in $H$. ingolfi (but not H. rostratus). Adult males of H. rostratus have the typical spine row on pleopod 1 (see Brökeland 2010).

However, the similarities between $H$. astraphes n. sp. and Antennuloniscus also include several other characters, which are found in many or even all species of Antennuloniscus. One of these is the small article 1 of antenna 2, formerly an autapomorphy of Antennuloniscus. The clypeus of $H$. astraphes $\mathbf{n}$. sp. is slightly prolonged (Fig. 3), but not as much as in most Antennuloniscus species. The armature of the pereopods is similar, with the distalmost ventral comb on the propodus drawn out towards the dactylus and one of the apical combs on the carpus composed of large spines. Within Antennuloniscus, preserved specimens often have antenna 1 directed posteriorly or laterally and therefore ventrally of antenna 2. A similar position of antenna 1 could be observed in some specimens of the new species. The proportions of articles 5 and 6 of antenna 2 are similar to those of most Antennuloniscus species. Like H. astraphes n. sp. many Antennuloniscus species have only two retinaculae on the maxilliped, in contrast to the usual three in most other haploniscids. Since no adult males of $H$. astraphes $\mathbf{n}$. sp. were found, similarities of pleopod 1 remain unclear. 


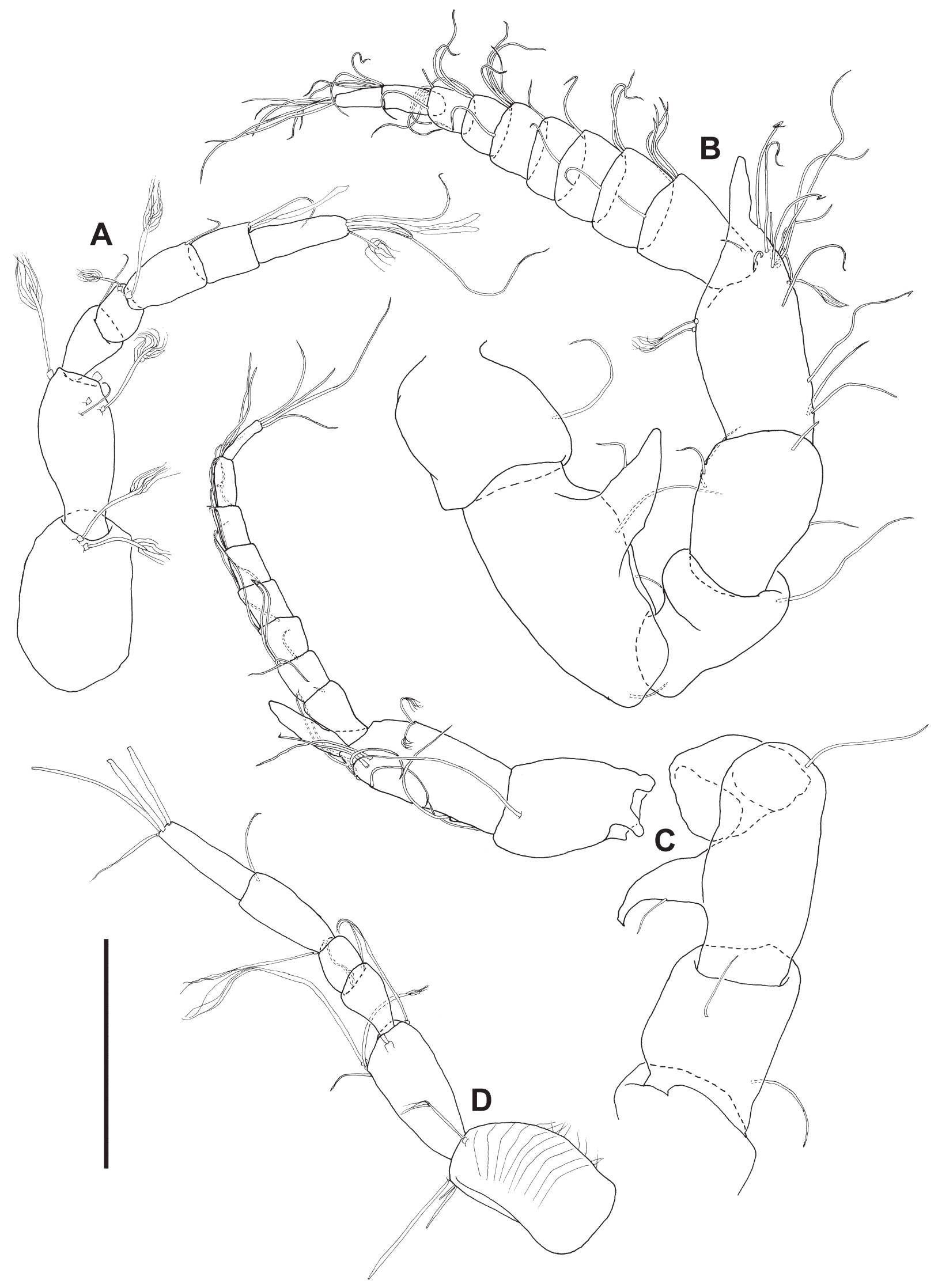

FIGURE 4. Haploniscus astraphes n. sp. male paratype, ZMH K-42502 (A, B), female paratype, ZMH K-42502 (C, D). A, antenna $1 ; \mathrm{B}$, antenna $2 ; \mathrm{C}$, antenna $2 ; \mathrm{D}$, antenna 1 . Scale bar $=0.1 \mathrm{~mm}$. 


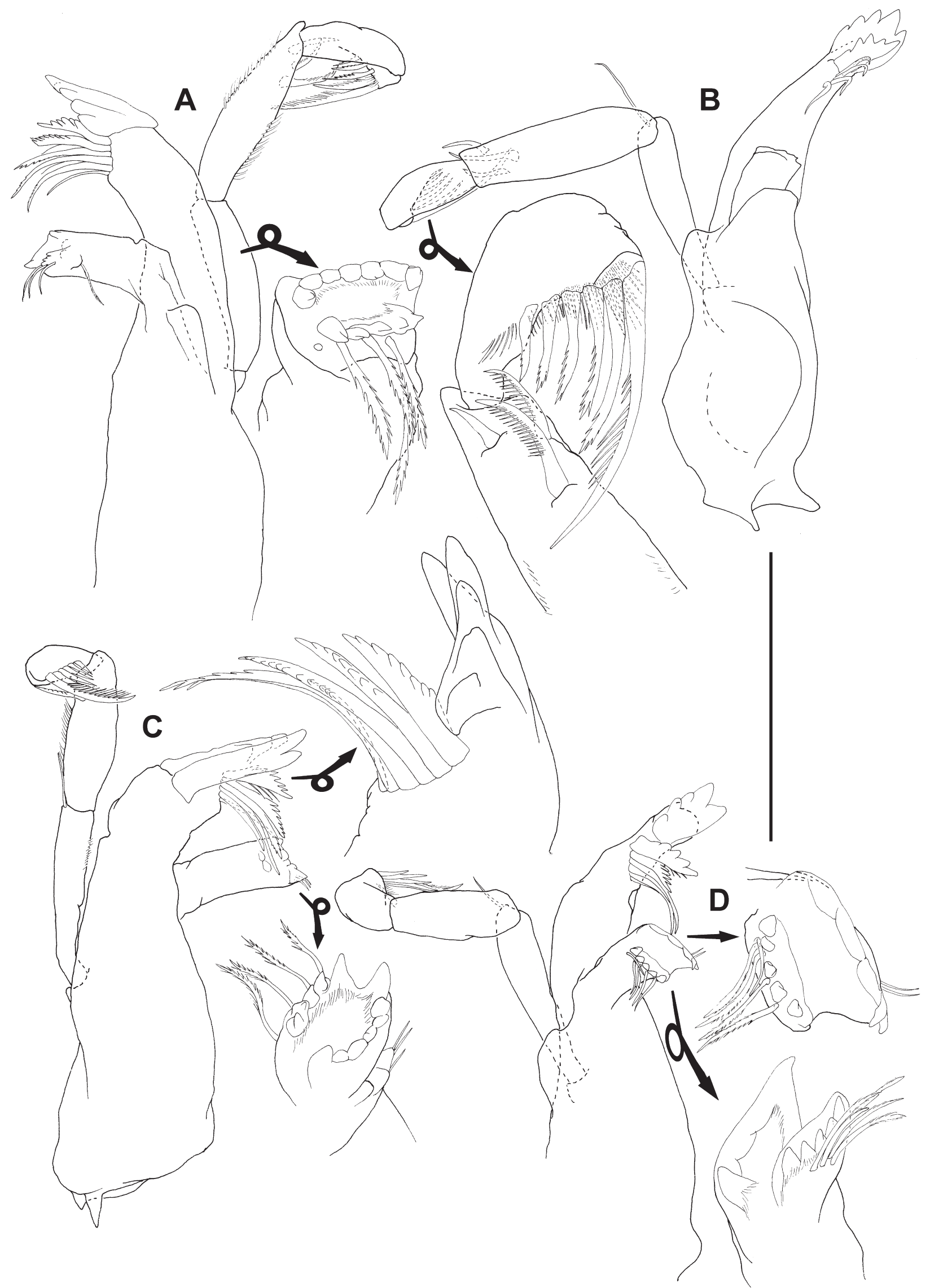

FIGURE 5. Haploniscus astraphes n. sp. male paratype, ZMH K-42502 (A, B), female paratype, ZMH K-42502 (C, D). A, right mandible; $\mathrm{B}$, left mandible; $\mathrm{C}$, right mandible; $\mathrm{D}$, left mandible. Scale bar $=0.1 \mathrm{~mm}$. 

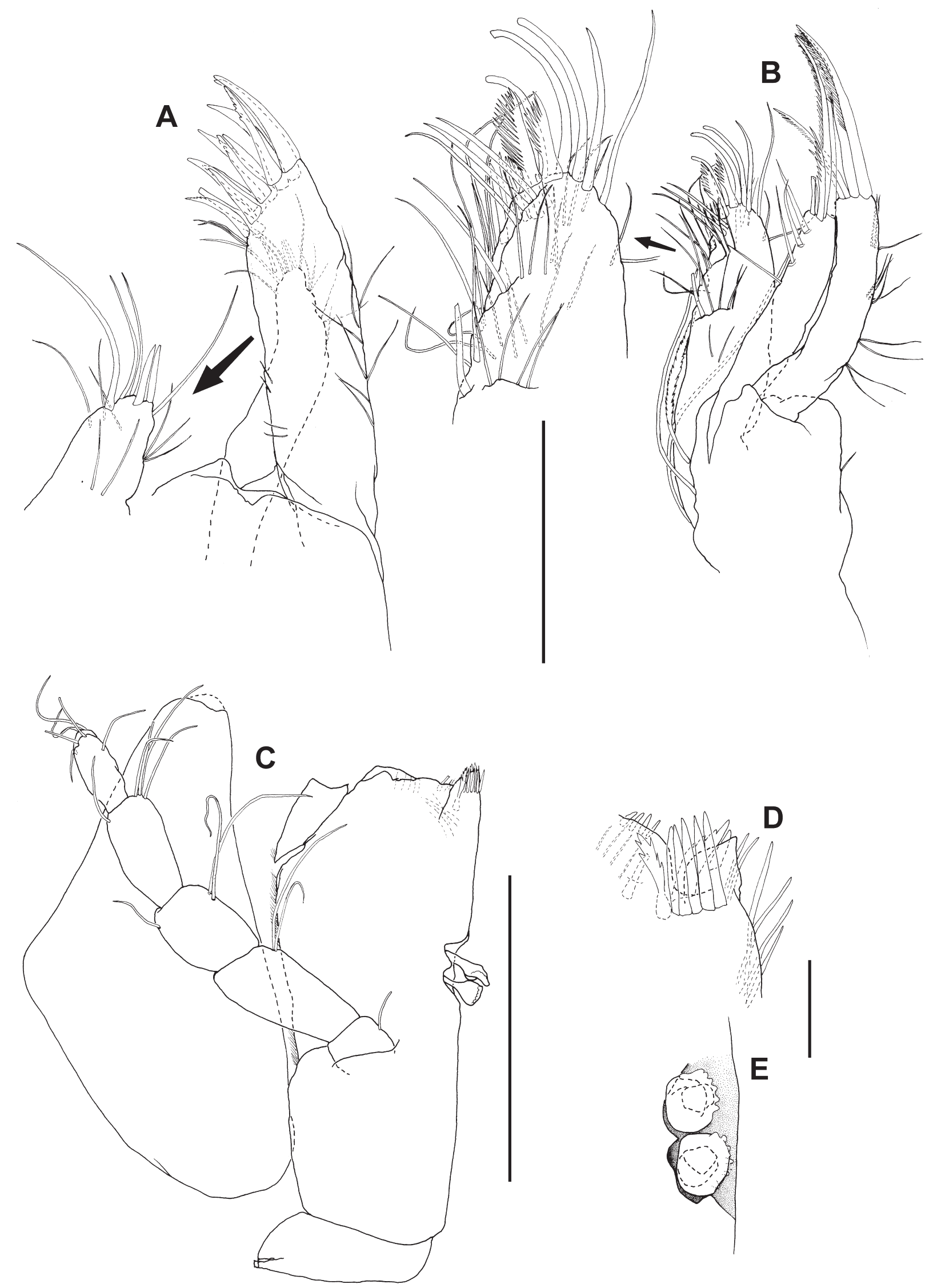

FIGURE 6. Haploniscus astraphes n. sp. male paratype, ZMH K-42502 (A, B and E, F), BIOICE female paratype, IMNH 10370 (C). A, maxilla 1; B, maxilla 2; C, maxilliped; D, maxilliped, detail of distomedial margin of endite; E, maxilliped, retinaculae. Scale bars: A, B $=0.5 \mathrm{~mm} ; C=0.1 \mathrm{~mm} ; \mathrm{D}, \mathrm{E}=0.01 \mathrm{~mm}$. 


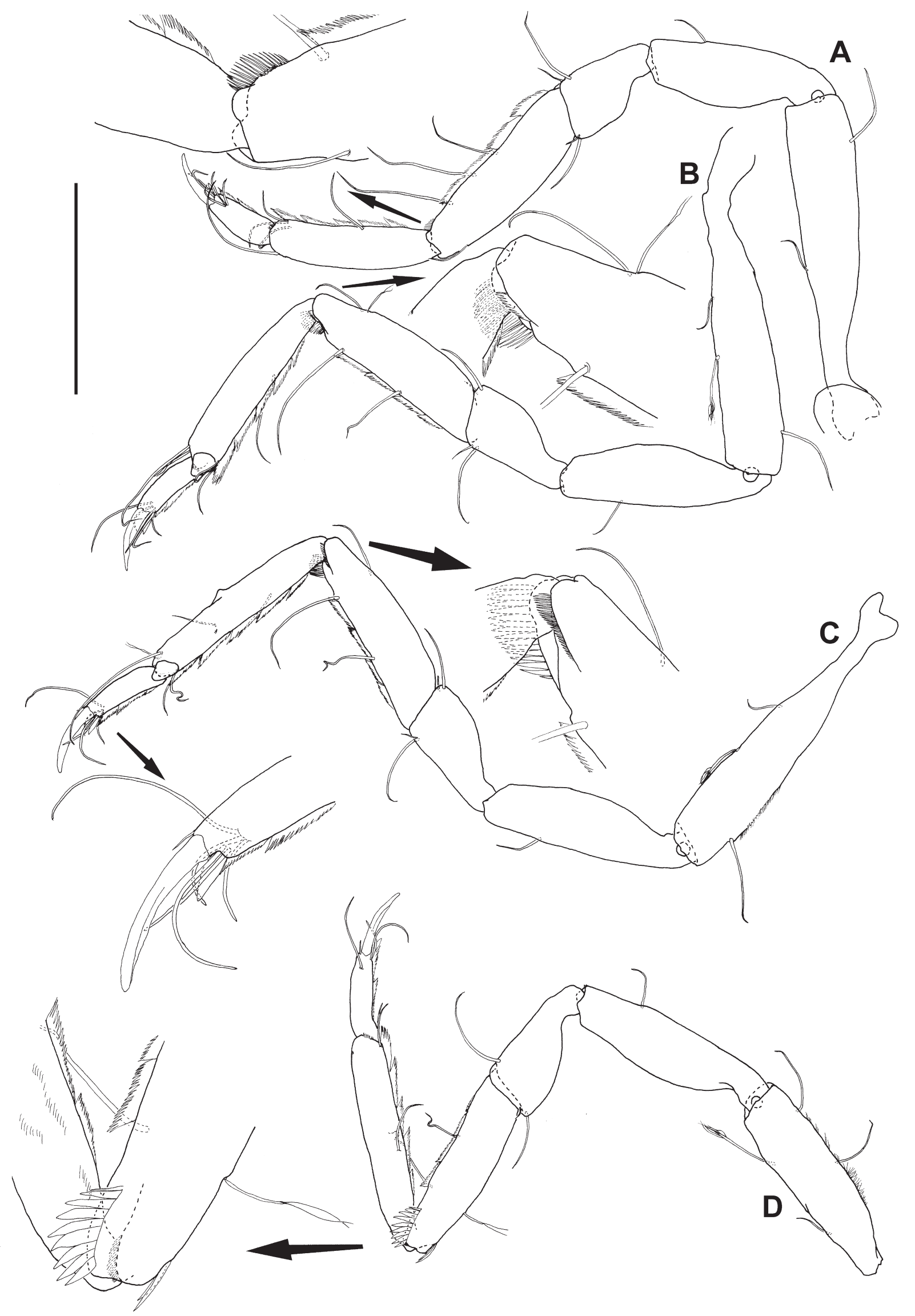

FIGURE 7. Haploniscus astraphes n. sp. male paratype, ZMH K-42502. A, pereopod 1; B, pereopod 2; C, pereopod 3; D, pereopod 4. Scale bar $=0.1 \mathrm{~mm}$. 


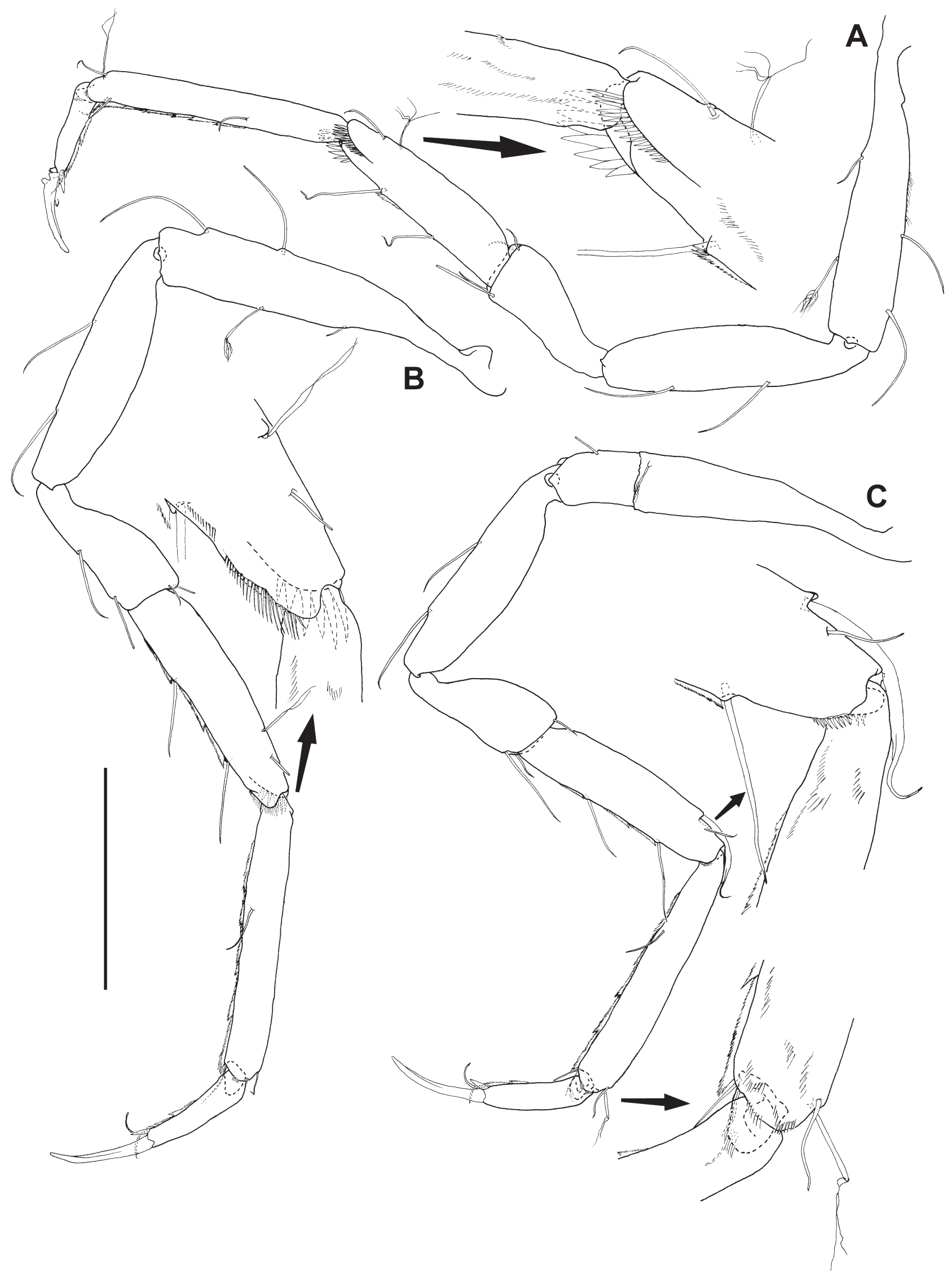

FIGURE 8. Haploniscus astraphes n. sp. male paratype, ZMH K-42502. A, pereopod 5; B, pereopod 6; C, pereopod 7. Scale bar $=0.1 \mathrm{~mm}$. 


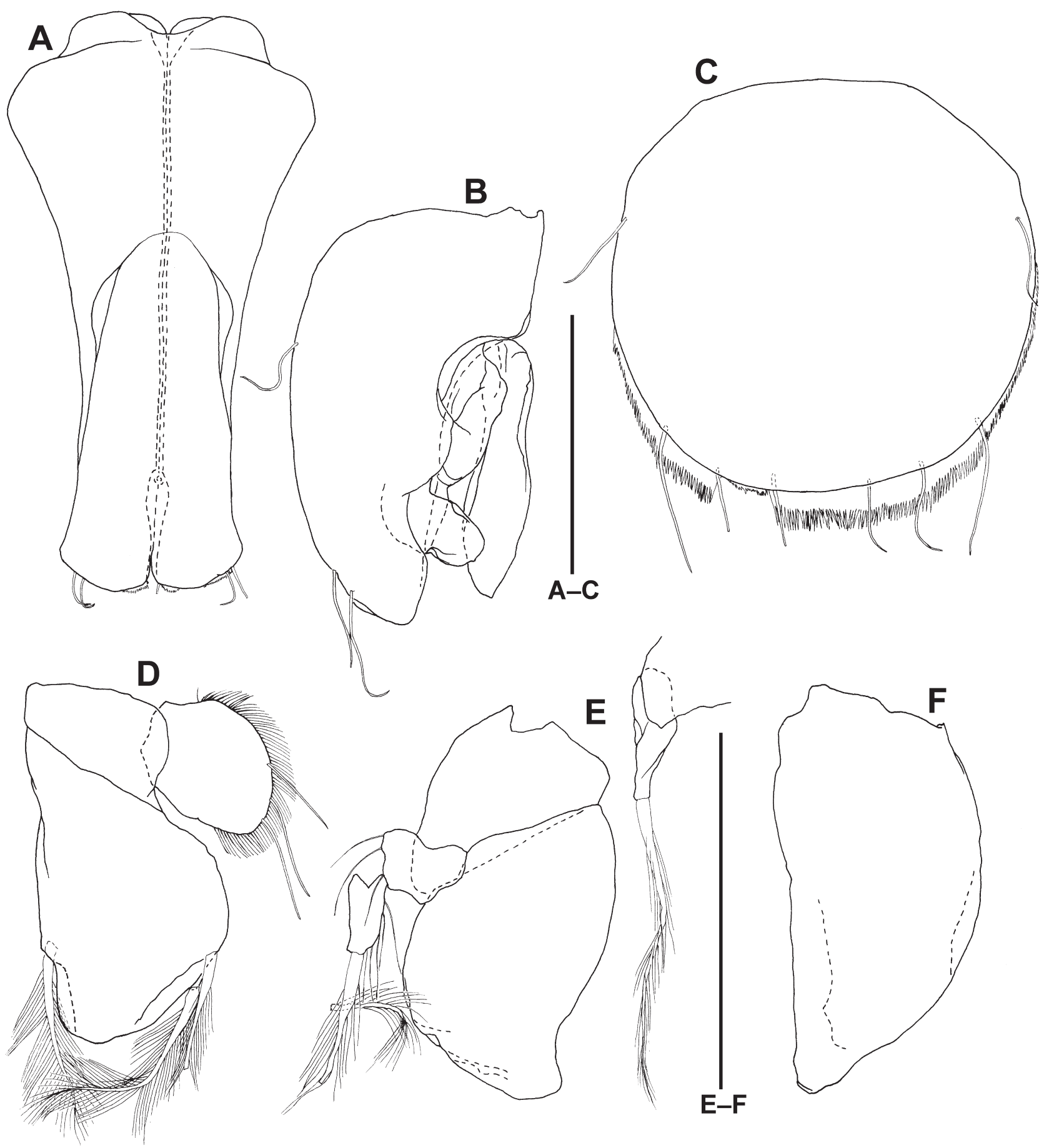

FIGURE 9. Haploniscus astraphes n. sp. male paratype, ZMH K-42502 (A, B), female paratype, ZMH K-42502 (C-F). A, pleopod 1; B, pleopod 2; C, pleopod 2; D, pleopod 3; E, pleopod 4; F, pleopod 5. Scale bars $=0.1 \mathrm{~mm}$.

H. pygmaeus Birstein, 1969 from the Romanche Trench (Birstein 1969) has very similar articles 5 and 6 of antenna 2 to that of $H$. astraphes n. sp., as well as the tooth on article 3, which is quite long in both species, but the two species differ distinctly in the shape of the head.

Since the most conspicuous character of Antennuloniscus, the fusion of antenna 2 articles 5 and 6 does not occur in $H$. astraphes $\mathbf{n}$. sp., it is placed in the genus Haploniscus. Considering the characters of H. astraphes $\mathbf{n}$. sp., $H$. ingolfi, $H$. pygmaeus and $H$. rostratus, as well as those of $A$. simplex, the only remaining apomorphy of Antennuloniscus is the fusion of articles 5 and 6 of antenna 2.

The only BIOICE station where Haploniscus astraphes n. sp. was sampled was located south of the 
Greenland-Iceland Ridge at depths of $1300 \mathrm{~m}$. The other localities are in the Guinea Basin, South Atlantic at more than $5000 \mathrm{~m}$ depth and the Angola Basin in more than $5600 \mathrm{~m}$ depth.

\section{Haploniscus aduncus Lincoln, 1985}

Haploniscus aduncus Lincoln, 1985b: 682, fig. 15a-b. —Kussakin 1988: 380-381, fig. 322. —Kavanagh 2009: 28.

Material examined. BIOICE stn. 2859, RV Bjarni Scemundsson, RP sled, 29 August 1995, 61ํ5022N, 165286W, $2270 \mathrm{~m}, 2.37^{\circ} \mathrm{C}, 1$ specimen (IMNH 36851); BIOICE stn. 2860, RV Bjarni Scemundsson, RP sled, 30 August 1995, $61^{\circ} 4343 \mathrm{~N}, 16^{\circ} 5748 \mathrm{~W}, 2295 \mathrm{~m}, 2.60^{\circ} \mathrm{C}, 1$ specimen (IMNH 36852); BIOICE stn. 3012, RV Bjarni Scemundsson, RP sled, 7 July 1997, 61 ${ }^{\circ} 2220 \mathrm{~N}, 15^{\circ} 1898 \mathrm{~W}, 2133 \mathrm{~m}, 3.29^{\circ} \mathrm{C}$, 3 specimens (IMNH 36853); BIOICE stn. 3075, RV Bjarni Scemundsson, RP sled, 13 July 1997, 62 ${ }^{\circ} 0019 \mathrm{~N}, 15^{\circ} 5997 \mathrm{~W}, 2192 \mathrm{~m}, 3.07^{\circ} \mathrm{C}, 1$ specimen (IMNH 36854); BIOICE stn. 3170, RV Bjarni Scemundsson, RP sled, 26 July 2000, 60 $3395 \mathrm{~N}, 22^{\circ} 0493 \mathrm{~W}, 2350 \mathrm{~m}, 2.81^{\circ} \mathrm{C}, 4$ specimens (IMNH 36855); BIOICE stn. 3176, RV Bjarni Samundsson, RP sled, 29 July 2000, 60 0203N, $22^{\circ} 2717$ W, 2537 m, 5 specimens (IMNH 36856); BIOICE stn. 3181, RV Bjarni Scemundsson, RP sled, 30 July 2000, 60 $5286 \mathrm{~N}, 26^{\circ} 4772 \mathrm{~W}, 1543 \mathrm{~m}, 3.53^{\circ} \mathrm{C}, 1$ specimen (IMNH 36857); BIOICE stn. 3570, RV Bjarni Scemundsson, RP sled, 4 September 2003, 63 ${ }^{\circ} 4271 \mathrm{~N}, 29^{\circ} 1036 \mathrm{~W}, 1819 \mathrm{~m}, 3.24^{\circ} \mathrm{C}, 3$ specimens (IMNH 36858 ); BIOICE stn. 3571, RV Bjarni Scemundsson, RP sled, 5 September 2003, 633022N, 29³840W, $2233 \mathrm{~m}, 3.14^{\circ} \mathrm{C}, 1$ specimen (IMNH 36859); BIOICE stn. 3576, RV Bjarni Scemundsson, RP sled, 6 September 2003, 63ำ1746N, $28^{\circ} 3439 \mathrm{~W}, 1744 \mathrm{~m}, 3.70^{\circ} \mathrm{C}, 11$ specimens (IMNH 36860 ).

Remarks. The only previous record of Haploniscus aduncus was from the type locality in the Iceland Basin $\left(60^{\circ} 7.1^{\prime}-60^{\circ} 8.3^{\prime} \mathrm{N}, 19^{\circ} 30.3^{\prime}-19^{\circ} 24.8^{\prime} \mathrm{W}, 2636-2646 \mathrm{~m}\right.$, Lincoln $\left.1985 \mathrm{~b}\right)$. The new records of $H$. aduncus were in the Iceland Basin, to the west and the north of the type locality, and additionally from the Irmiger Basin, at depths between 1543 and $2537 \mathrm{~m}$ (Table 1, Fig. 10B).

\section{Haploniscus ampliatus Lincoln, 1985}

Haploniscus ampliatus Lincoln, 1985b: 663-666, figs 3-4.-Kussakin 1988: 371-373, fig. 317.—Kavanagh 2009: 28-29.

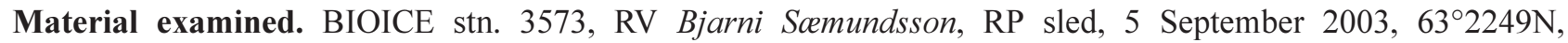
$29^{\circ} 5485 \mathrm{~W}, 2359 \mathrm{~m}, 3.06^{\circ} \mathrm{C}, 4$ specimens (IMNH 36861 ).

Remarks. Haploniscus ampliatus was collected only at a single station west of Iceland in the Irmiger Basin at $2359 \mathrm{~m}$ (Fig. 10A, triangle). Previous records of H. ampliatus are from a cluster of stations off the northern edge of the Porcupine Bank and to the north in the Iceland Basin, at 2636-2925 m (Lincoln 1985b).

\section{Haploniscus angustus Lincoln, 1985}

Haploniscus angustus Lincoln, 1985b: 676-679, figs 11-12.-Kussakin 1988: 390-392, fig. 329._Kavanagh $2009: 29$.

Material examined. BIOICE stn. 2579, RV Håkon Mosby, RP sled, 15 July 1993, 670575N. 24²4327W, 996 m, $0.58^{\circ} \mathrm{C}, 1$ specimen (IMNH 36862). BIOICE stn. 2856, RV Bjarni Scemundsson, RP sled, 29 August 1995, $62^{\circ} 2050 \mathrm{~N}, 16^{\circ} 5930 \mathrm{~W}, 2074 \mathrm{~m}, 2.34^{\circ} \mathrm{C}, 3$ specimens (IMNH 36863); BIOICE stn. 2859, RV Bjarni Scemundsson, RP sled, 29 August 1995, 61 ${ }^{\circ} 5022 \mathrm{~N}, 16^{\circ} 5286 \mathrm{~W}, 2270 \mathrm{~m}, 2.37^{\circ} \mathrm{C}, 1$ specimen (IMNH 36864); BIOICE stn. 3659, RV Håkon Mosby, RP sled, 24 July 2004, 670761N, $19^{\circ} 3638 \mathrm{~W}, 806 \mathrm{~m},-0.53^{\circ} \mathrm{C}, 2$ specimens (IMNH 36865 ).

Remarks. Only females of $H$. angustus were found, which occurred on both sides of the GIF Ridge (Fig. 10C). The species has earlier been reported from several stations in the northern part of the Rockall Trough and the Porcupine Sea-Bight (Lincoln 1985b; Kavanagh 2009). Svavarsson (1988) reported specimens of $H$. ingolfi from the Nordic Seas lacking a terminal process on the sixth article of antenna 2, which is one of the characteristics of $H$. ingolfi. Those specimens may possibly belong to the present species which does not have such process, rather than belonging to $H$. ingolfi. 


\section{Haploniscus bicuspis (Sars, 1877)}

Nannoniscus bicuspis Sars, 1877: 352.-Sars 1885: 122, pl. 10: figs 31-45.

Haploniscus bicuspis - Richardson 1908: 75.-Hansen 1916: 29-30, pl. 2, figs 2a-1.—Gurjanova 1932: 28.—Wolff 1962: 58, 208-210, 226 figs 25, 138-139, 144.- Lincoln 1985b: 659-662, figs 1-2.-Kussakin 1988: 365-370, figs 312-315.Svavarsson 1988: 85-86.-Brökeland and Wägele 2004: 2-4, figs 1-12.-Brökeland and Wägele 2004: 237, fig. 1 (Electronic supplement, 2-4, figs 1-12).-Kavanagh 2009: 29.

Haploniscus spinifer Hansen, 1916: 31 (part.).

Material examined. BIOICE stn. 2003, RV Håkon Mosby, RP sled, 19 July 1991, 652098N, 133657W, 64 m, $5.17^{\circ} \mathrm{C}, 3$ specimens (IMNH 36866); BIOICE stn. 2010, RV Håkon Mosby, RP sled, 20 July 1991, 653022N, $11^{\circ} 2345 \mathrm{~W}, 619 \mathrm{~m},-0.36^{\circ} \mathrm{C}, 18$ specimens (IMNH 36867); BIOICE stn. 2011, RV Håkon Mosby, RP sled, 20 July

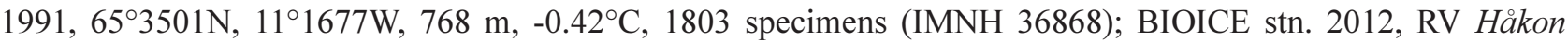
Mosby, Sneli sled, 20 July 1991, 65 ${ }^{\circ} 3508 \mathrm{~N}, 11^{\circ} 1662 \mathrm{~W}, 769 \mathrm{~m},-0.42^{\circ} \mathrm{C}, 13$ specimens (IMNH 36869); BIOICE stn. 2014, RV Håkon Mosby, RP sled, 20 July 1991, 654143N, $10^{\circ} 5998 \mathrm{~W}, 978 \mathrm{~m},-0.41^{\circ} \mathrm{C}, 4382$ specimens (IMNH 36870); BIOICE stn. 2015, RV Håkon Mosby, RP sled, 21 July 1991, 655907N, 104391W, 1195 m, $0.79^{\circ} \mathrm{C}, 50$ specimens (IMNH 36871); BIOICE stn. 2018, RV Håkon Mosby, RP sled, 21 July 1991, 66 $6^{\circ} 3397 \mathrm{~N}$, $11^{\circ} 1349 \mathrm{~W}, 1390 \mathrm{~m},-0.82^{\circ} \mathrm{C}, 26$ specimens (IMNH 36872); BIOICE stn. 2019, RV Håkon Mosby, RP sled, 21 July $1991,66^{\circ} 3317 \mathrm{~N}, 12^{\circ} 1156 \mathrm{~W}, 1253 \mathrm{~m},-0.86^{\circ} \mathrm{C}, 718$ specimens (IMNH 36873); BIOICE stn. 2020, RV Håkon Mosby, Sneli sled, 22 July $1991,66^{\circ} 3740 \mathrm{~N}, 12^{\circ} 0875 \mathrm{~W}, 1314 \mathrm{~m},-0.86^{\circ} \mathrm{C}, 1$ specimen (IMNH 36874); BIOICE stn. 2021, RV Håkon Mosby, Sneli sled, 22 July 1991, 66³829N, 12³531W, 1018 m, 5 specimens (IMNH 36875); BIOICE stn. 2023, RV Håkon Mosby, Sneli sled, 22 July 1991, 66 $3323 \mathrm{~N}, 13^{\circ} 1526 \mathrm{~W}, 294 \mathrm{~m}, 1.41^{\circ} \mathrm{C}, 64$ specimens (IMNH 36876); BIOICE stn. 2024, RV Håkon Mosby, RP sled, 22 July 1991, 66 $3222 \mathrm{~N}, 13^{\circ} 1700 \mathrm{~W}$, $317 \mathrm{~m}, 1.41^{\circ} \mathrm{C}, 1$ specimen (IMNH 36877); BIOICE stn. 2025, RV Håkon Mosby, RP sled, 22 July 1991, $67^{\circ} 0077 \mathrm{~N}, 13^{\circ} 3208 \mathrm{~W}, 781 \mathrm{~m},-0.54^{\circ} \mathrm{C}, 52$ specimens (IMNH 36878); BIOICE stn. 2027, RV Håkon Mosby, RP sled, 23 July $1991,67^{\circ} 1182 \mathrm{~N}, 13^{\circ} 2058 \mathrm{~W}, 1648 \mathrm{~m},-0.76^{\circ} \mathrm{C}, 116$ specimens (IMNH 36879 ); BIOICE stn. 2030 , RV Håkon Mosby, RP sled, 23 July 1991, 67 $0029 \mathrm{~N}, 13^{\circ} 2585 \mathrm{~W}, 831 \mathrm{~m},-0.55^{\circ} \mathrm{C}, 766$ specimens (IMNH 36880); BIOICE stn. 2033, RV Håkon Mosby, RP sled, 23 July 1991, 66 $6^{\circ} 5481 \mathrm{~N}, 13^{\circ} 3065 \mathrm{~W}, 552 \mathrm{~m},-0.54^{\circ} \mathrm{C}, 35$ specimens (IMNH 36881); BIOICE stn. 2076, RV Håkon Mosby, Shipek grab, 30 July 1992, 67¹260N, $17^{\circ} 3381$ W, 622 m, $0.42^{\circ} \mathrm{C}, 2$ specimens (IMNH 36882); BIOICE stn. 2082, RV Håkon Mosby, Sneli sled, 4 July 1992, 67²203N, $17^{\circ} 2433 \mathrm{~W}, 894 \mathrm{~m},-0.55^{\circ} \mathrm{C}, 3$ specimens (IMNH 36883); BIOICE stn. 2088, RV Håkon Mosby, RP sled, 4 July $1992,67^{\circ} 1432 \mathrm{~N}, 17^{\circ} 5141 \mathrm{~W}, 617 \mathrm{~m},-0.44^{\circ} \mathrm{C}, 7$ specimens (IMNH 36884); BIOICE stn. 2090, RV Håkon Mosby, RP sled, 5 July $1992,67^{\circ} 1334 \mathrm{~N}, 17^{\circ} 4894 \mathrm{~W}, 539 \mathrm{~m},-0.44^{\circ} \mathrm{C}, 36$ specimens (IMNH 36885); BIOICE stn. 2093, RV Håkon Mosby, Sneli sled, 5 July 1992, $67^{\circ} 1185 \mathrm{~N}, 17^{\circ} 4530 \mathrm{~W}, 407 \mathrm{~m},-0.31^{\circ} \mathrm{C}, 8$ specimens (IMNH 36886 );

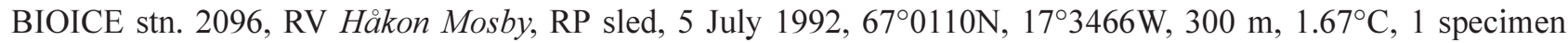
(IMNH 36887); BIOICE stn. 2100, RV Håkon Mosby, RP sled, 6 July 1992, 680006N, $19^{\circ} 2526 \mathrm{~W}, 1141 \mathrm{~m}$, $0.58^{\circ} \mathrm{C}, 32$ specimens (IMNH 36888); BIOICE stn. 2102, RV Håkon Mosby, Sneli sled, 6 July 1992, 675986N, $19^{\circ} 2629 \mathrm{~W}, 1146 \mathrm{~m},-0.58^{\circ} \mathrm{C}, 1$ specimen (IMNH 36889); BIOICE stn. 2103, RV Håkon Mosby, Sneli sled, 6 July $1992,67^{\circ} 5004 \mathrm{~N}, 19^{\circ} 3287 \mathrm{~W}, 905 \mathrm{~m},-0.55^{\circ} \mathrm{C}, 4$ specimens (IMNH 36890); BIOICE stn. 2110, RV Håkon Mosby, Sneli sled, 6 July $1992,67^{\circ} 4571 \mathrm{~N}, 1^{\circ} 2951 \mathrm{~W}, 762 \mathrm{~m},-0.53^{\circ} \mathrm{C}, 1$ specimen (IMNH 36891 ); BIOICE stn. 2132 , RV Håkon Mosby, RP sled, 8 July 1992, 66 $4465 \mathrm{~N}, 18^{\circ} 5530 \mathrm{~W}, 492 \mathrm{~m}, 0.12^{\circ} \mathrm{C}, 5$ specimens (IMNH 36892 ); BIOICE stn. 2134, RV Håkon Mosby, Sneli sled, 8 July 1992, 66 ${ }^{\circ} 4446 \mathrm{~N}, 18^{\circ} 5493 \mathrm{~W}, 504 \mathrm{~m}, 0.12^{\circ} \mathrm{C}, 2$ specimens (IMNH

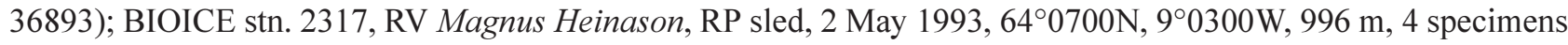
(IMNH 36894); BIOICE stn. 2334, RV Magnus Heinason, RP sled, 4 May 1993, 624300N, $12^{\circ} 4300 \mathrm{~W}, 803$ m, 2

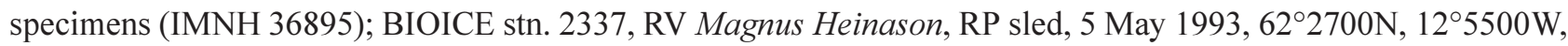
1099 m, 19 specimens (IMNH 36896); BIOICE stn. 2364, RV Magnus Heinason, RP sled, 8 May 1993, 643500N, $10^{\circ} 0300 \mathrm{~W}, 605$ m, 63 specimens (IMNH 36897); BIOICE stn. 2366, RV Magnus Heinason, Sneli sled, 8 May 1993, 643900N, 94700W, 675 m, 1 specimen (IMNH 36898); BIOICE stn. 2369, RV Magnus Heinason, Sneli sled, 8 May 1993, 644000N, 9³400W, 920 m, 1 specimen (IMNH 36899); BIOICE stn. 2403, RV Bjarni

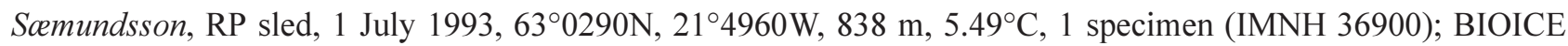
stn. 2406, RV Bjarni Scemundsson, RP sled, 1 July 1993, 62 $5920 \mathrm{~N}, 21^{\circ} 4700 \mathrm{~W}, 934 \mathrm{~m}, 4.57^{\circ} \mathrm{C}, 79$ specimens

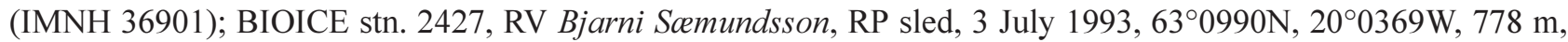
$5.50^{\circ} \mathrm{C}, 1$ specimen (IMNH 36902); BIOICE stn. 2475, RV Bjarni Scemundsson, RP sled, 5 July 1993, 630ㄴ20N, 
$21^{\circ} 3490 \mathrm{~W}, 842 \mathrm{~m}, 5.54^{\circ} \mathrm{C}, 42$ specimens (IMNH 36903); BIOICE stn. 2575, RV Håkon Mosby, RP sled, 15 July $1993,67^{\circ} 0964 \mathrm{~N}, 24^{\circ} 1931 \mathrm{~W}, 800 \mathrm{~m},-0.53^{\circ} \mathrm{C}, 96$ specimens (IMNH 36904); BIOICE stn. 2578, RV Håkon Mosby, Sneli sled, 15 July $1993,67^{\circ} 0822 \mathrm{~N}, 2^{\circ} 3666 \mathrm{~W}, 1002 \mathrm{~m},-0.58^{\circ} \mathrm{C}, 4$ specimens (IMNH 36905 ); BIOICE stn. 2579 , RV Håkon Mosby, RP sled, 15 July 1993, 67 $0575 \mathrm{~N}, 24^{\circ} 4327,996 \mathrm{~m},-0.58^{\circ} \mathrm{C}, 214$ specimens (IMNH 36906); BIOICE stn. 2581, RV Håkon Mosby, RP sled, 16 July $1993,67^{\circ} 4320 \mathrm{~N}, 22^{\circ} 3446 \mathrm{~N}, 719 \mathrm{~m},-0.50^{\circ} \mathrm{C}, 4$ specimens (IMNH 36907); BIOICE stn. 2585, RV Håkon Mosby, RP sled, 16 July 1993, 67²477N, $22^{\circ} 2386 \mathrm{~W}, 450 \mathrm{~m}$, $0.46^{\circ} \mathrm{C}, 1$ specimen (IMNH 36908); BIOICE stn. 2591, RV Håkon Mosby, RP sled, 16 July 1993, 67²53N, $22^{\circ} 2546 \mathrm{~W}, 333 \mathrm{~m}, 0.12^{\circ} \mathrm{C}, 19$ specimens (IMNH 36909); BIOICE stn. 2627, RV Håkon Mosby, RP sled, 12 July 1994, 67²543N, $16^{\circ} 0973 \mathrm{~W}, 748 \mathrm{~m},-0.56^{\circ} \mathrm{C}, 26$ specimens (IMNH 36910); BIOICE stn. 2634, RV Håkon Mosby, Sneli sled, 12 July 1994, 67 $2976 \mathrm{~N}, 15^{\circ} 4785 \mathrm{~W}, 795 \mathrm{~m},-0.57^{\circ} \mathrm{C}, 1$ specimen (IMNH 36911); BIOICE stn. 2638, RV Håkon Mosby, RP sled, 13 July 1994, 67²4868N, $15^{\circ} 3018 \mathrm{~W}, 1009$ m, $-0.59^{\circ} \mathrm{C}, 67$ specimens (IMNH 36912); BIOICE stn. 2640, RV Håkon Mosby, Sneli sled, 13 July 1994, 67 5571N, $15^{\circ} 2181 \mathrm{~W}, 1097 \mathrm{~m},-0.61^{\circ} \mathrm{C}, 2$ specimens (IMNH 36913); BIOICE stn. 2642, RV Håkon Mosby, RP sled, 13 July 1994, 675591N, 15²129W, 1098 m, - $0.61^{\circ} \mathrm{C}, 117$ specimens (IMNH 36914); BIOICE stn. 2644, RV Håkon Mosby, RP sled, 13 July 1994, $68^{\circ} 0115 \mathrm{~N}, 15^{\circ} 1467 \mathrm{~W}, 1202 \mathrm{~m},-0.64^{\circ} \mathrm{C}, 4$ specimens (IMNH 36915); BIOICE stn. 2647, RV Håkon Mosby, Sneli sled, 13 July 1994, $68^{\circ} 0492 \mathrm{~N}, 15^{\circ} 1933 \mathrm{~W}, 1304 \mathrm{~m},-0.72^{\circ} \mathrm{C}, 2$ specimens (IMNH 36916); BIOICE stn. 2648, RV Håkon Mosby, RP sled, 14 July 1994, 68 $0509 \mathrm{~N}, 15^{\circ} 1865 \mathrm{~W}, 1306 \mathrm{~m},-0.72^{\circ} \mathrm{C}, 76$ specimens (IMNH 36917); BIOICE stn. 2652, RV Håkon Mosby, RP sled, 14 July 1994, 67³597N, $15^{\circ} 0742 \mathrm{~W}, 910 \mathrm{~m},-0.57^{\circ} \mathrm{C}, 5$ specimens (IMNH 36918); BIOICE stn. 2655, RV Håkon Mosby, RP sled, 14 July 1994, 67¹800N, 145ㄱㅇ, 549 m, $0.55^{\circ} \mathrm{C}, 13$ specimens (IMNH 36919); BIOICE stn. 2693, RV Bjarni Scemundsson, Agassiz trawl, 31 August 1994, $64^{\circ} 2700 \mathrm{~N}, 28^{\circ} 1000 \mathrm{~W}, 1100 \mathrm{~m}, 3.72^{\circ} \mathrm{C}, 1$ specimen (IMNH 36920); BIOICE stn. 2700, RV Bjarni Scemundsson, Sneli sled, 2 September $1994,64^{\circ} 0560 \mathrm{~N}, 27^{\circ} 5000 \mathrm{~W}, 1105 \mathrm{~m}, 3.84^{\circ} \mathrm{C}, 1$ specimen (IMNH 36921 ); BIOICE stn. 2743, RV Håkon Mosby, Sneli sled, 30 July 1995, 670495N, 20²841 W, 729 m, 2 specimens (IMNH 36922); BIOICE stn. 2747, RV Håkon Mosby, Sneli sled, 31 July 1995, 670639N, 20 $4715 \mathrm{~W}, 778$ m, $-0.58^{\circ} \mathrm{C}, 3$ specimens (IMNH 36923); BIOICE stn. 2754, RV Håkon Mosby, RP sled, 31 July 1995, 675504N, 19¹897W, $1023 \mathrm{~m},-0.61^{\circ} \mathrm{C}, 11$ specimens (IMNH 36924); BIOICE stn. 2755, RV Håkon Mosby, Sneli sled, 31 July 1995, $67^{\circ} 5513 \mathrm{~N}, 19^{\circ} 1900 \mathrm{~W}, 1024 \mathrm{~m},-0.61^{\circ} \mathrm{C}, 2$ specimens (IMNH 36925); BIOICE stn. 2756, RV Håkon Mosby, Triangle dredge, 1 August $1995,67^{\circ} 5514 \mathrm{~N}, 18^{\circ} 1880 \mathrm{~W}, 610 \mathrm{~m},-0.39^{\circ} \mathrm{C}, 4$ specimens (IMNH 36926 ); BIOICE stn. 2758, RV Håkon Mosby, Triangle dredge, 1 August 1995, 67 $5478 \mathrm{~N}, 18^{\circ} 0830 \mathrm{~W}, 786 \mathrm{~m},-0.39^{\circ} \mathrm{C}, 7$ specimens

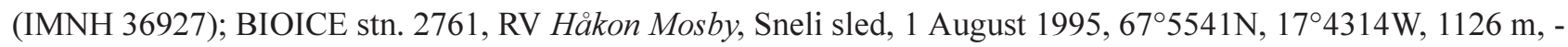
$0.53^{\circ} \mathrm{C}$, 1 specimen (IMNH 36928); BIOICE stn. 2762, RV Håkon Mosby, RP sled, 1 August 1995, 67 5548N, $17^{\circ} 4226 \mathrm{~W}, 1130 \mathrm{~m},-0.53^{\circ} \mathrm{C}, 305$ specimens (IMNH 36929); BIOICE stn. 2765, RV Håkon Mosby, RP sled, 1 August 1995, $68^{\circ} 0630 \mathrm{~N}, 17^{\circ} 3205 \mathrm{~W}, 1220 \mathrm{~m},-0.52^{\circ} \mathrm{C}, 37$ specimens (IMNH 36930); BIOICE stn. 2769, RV Håkon Mosby, Triangle dredge, 2 August 1995, 68 ${ }^{\circ} 3518 \mathrm{~N}, 16^{\circ} 5623 \mathrm{~W}, 519 \mathrm{~m},-0.37^{\circ} \mathrm{C}, 1$ specimen (IMNH 36931); BIOICE stn. 2773, RV Håkon Mosby, RP sled, 3 August 1995, $69^{\circ} 1489 \mathrm{~N}, 14^{\circ} 1652,1629 \mathrm{~m},-0.86^{\circ} \mathrm{C}, 3$ specimens (IMNH 36932); BIOICE stn. 2776, RV Håkon Mosby, Sneli sled, 3 August 1995, 68³610N, $14^{\circ} 3992 \mathrm{~W}, 1553 \mathrm{~m},-0.78^{\circ} \mathrm{C}, 1$ specimen (IMNH 36933); BIOICE stn. 2777, RV Håkon Mosby, RP sled, 3 August $1995,68^{\circ} 3680 \mathrm{~N}, 14^{\circ} 4070 \mathrm{~W}, 1556 \mathrm{~m},-0.78^{\circ} \mathrm{C}, 19$ specimens (IMNH 36934); BIOICE stn. 2779, RV Håkon Mosby, RP sled, 4 August 1995, 68 $2042 \mathrm{~N}, 15^{\circ} 4669 \mathrm{~W}, 1413 \mathrm{~m},-0.74^{\circ} \mathrm{C}, 16$ specimens (IMNH 36935); BIOICE

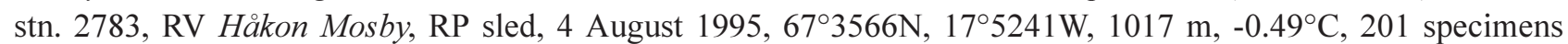
(IMNH 36936); BIOICE stn. 2786, RV Håkon Mosby, RP sled, 5 August 1995, 67²456N, $18^{\circ} 1430 \mathrm{~W}, 693 \mathrm{~m}$, $0.41^{\circ} \mathrm{C}, 81$ specimens (IMNH 36937); BIOICE stn. 2787, RV Håkon Mosby, RP sled, 5 August 1995, 67²136N, $18^{\circ} 2004 \mathrm{~W}, 561 \mathrm{~m},-0.29^{\circ} \mathrm{C}, 4$ specimens (IMNH 36938); BIOICE stn. 2789, RV Håkon Mosby, Sneli sled, 5 August $1995,67^{\circ} 1865 \mathrm{~N}, 18^{\circ} 2312 \mathrm{~W}, 540 \mathrm{~m},-0.29^{\circ} \mathrm{C}, 3$ specimens (IMNH 36939); BIOICE stn. 2844, RV Bjarni

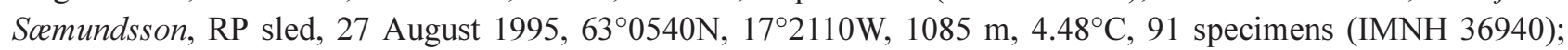
BIOICE stn. 2846, RV Bjarni Scemundsson, RP sled, 27 August 1995, 62 $5900 \mathrm{~N}, 17^{\circ} 5010 \mathrm{~W}, 947 \mathrm{~m}, 3.48^{\circ} \mathrm{C}, 5$ specimens (IMNH 36941); BIOICE stn. 2849, RV Bjarni Scemundsson, RP sled, 27 August 1995, 624982N, $18^{\circ} 0042 \mathrm{~W}, 976 \mathrm{~m}, 3.26^{\circ} \mathrm{C}, 1$ specimen (IMNH 36942); BIOICE stn. 2853, RV Bjarni Scemundsson, RP sled, 28 August 1995, 62 $2^{\circ} 030 \mathrm{~N}, 16^{\circ} 5820 \mathrm{~W}, 1833 \mathrm{~m}, 2.40^{\circ} \mathrm{C}, 2$ specimens (IMNH 36943); BIOICE stn. 2901, RV Bjarni

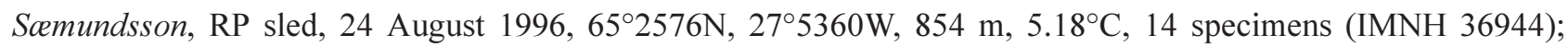
BIOICE stn. 2903, RV Bjarni Scemundsson, Sneli sled, 24 August 1996, $65^{\circ} 2280 \mathrm{~N}, 28^{\circ} 2063 \mathrm{~W}, 1066 \mathrm{~m}, 4.78^{\circ} \mathrm{C}, 1$ specimen (IMNH 36945); BIOICE stn. 2904, RV Bjarni Scemundsson, RP sled, 24 August 1996, 65²327N, 
$28^{\circ} 2127 \mathrm{~W}, 1057 \mathrm{~m}, 4.78^{\circ} \mathrm{C}, 6$ specimens (IMNH 36946); BIOICE stn. 2909, RV Bjarni Scemundsson, RP sled, 25 August 1996, $65^{\circ} 1561 \mathrm{~N}, 28^{\circ} 5015 \mathrm{~W}, 1300 \mathrm{~m}, 3.96^{\circ} \mathrm{C}, 9$ specimens (IMNH 36947); BIOICE stn. 2912, RV Bjarni Scemundsson, RP sled, 25 August 1996, 65 $1101 \mathrm{~N}, 29^{\circ} 0418 \mathrm{~W}, 1456 \mathrm{~m}, 3.90^{\circ} \mathrm{C}, 38$ specimens (IMNH 36948); BIOICE stn. 2918, RV Bjarni Scemundsson, Sneli sled, 26 August 1996, 651257N, $29^{\circ} 1455 \mathrm{~W}, 1539 \mathrm{~m}, 3.22^{\circ} \mathrm{C}, 1$ specimen (IMNH 36949); BIOICE stn. 3072, RV Bjarni Scemundsson, RP sled, 12 July 1997, 615365N, $15^{\circ} 0815 \mathrm{~W}, 2082 \mathrm{~m}, 2.83^{\circ} \mathrm{C}, 28$ specimens (IMNH 36950); BIOICE stn. 3115, RV Håkon Mosby, RP sled, 22 August 1999, $67^{\circ} 4218 \mathrm{~N}, 19^{\circ} 4755 \mathrm{~W}, 611 \mathrm{~m},-0.57^{\circ} \mathrm{C}, 2$ specimens (IMNH 36951); BIOICE stn. 3127, RV Håkon Mosby, RP sled, 23 August 1999, 684357N, 16³467W, 1715 m, 33 specimens (IMNH 36952); BIOICE stn. 3136, RV Håkon Mosby, RP sled, 24 August 1999, 68 ${ }^{\circ} 1527 \mathrm{~N}, 20^{\circ} 0108 \mathrm{~W}, 1112$ m, 12 specimens (IMNH 36953); BIOICE stn. 3140, RV Håkon Mosby, RP sled, 25 August 1999, 67 $5190 \mathrm{~N}, 22^{\circ} 1489 \mathrm{~W}, 768 \mathrm{~m},-0.46^{\circ} \mathrm{C}, 11$ specimens (IMNH 36954); BIOICE stn. 3143, RV Håkon Mosby, RP sled, 25 August 1999, 67³076N, $24^{\circ} 1003 \mathrm{~W}$, 1012 m, 79 specimens (IMNH 36955); BIOICE stn. 3149, RV Håkon Mosby, Sneli sled, 26 August 1999, $66^{\circ} 5263 \mathrm{~N}, 26^{\circ} 5603 \mathrm{~W}, 434 \mathrm{~m}, 0.06^{\circ} \mathrm{C}, 2$ specimens (IMNH 36956); BIOICE stn. 3163, RV Bjarni Scemundsson, Agassiz trawl, 26 July $2000,61^{\circ} 4150 \mathrm{~N}, 22^{\circ} 5810 \mathrm{~W}, 1743 \mathrm{~m}, 2.79^{\circ} \mathrm{C}, 45$ specimens (IMNH 36957 ); BIOICE stn. 3177, RV Bjarni Scemundsson, RP sled, 29 July 2000, 604491N, 234272W, 1951 m, 4 specimens (IMNH 36958); BIOICE stn. 3181, RV Bjarni Scemundsson, RP sled, 30 July 2000, $60^{\circ} 5286 \mathrm{~N}, 26^{\circ} 4772 \mathrm{~W}, 1543 \mathrm{~m}, 3.53^{\circ} \mathrm{C}, 9$ specimens (IMNH 36959); BIOICE stn. 3189, RV Bjarni Scemundsson, Agassiz trawl, 1 August 2000, 62 $0863 \mathrm{~N}$, $26^{\circ} 3817 \mathrm{~W}, 950 \mathrm{~m}, 5.21^{\circ} \mathrm{C}, 4$ specimens (IMNH 36960); BIOICE stn. 3195, RV Håkon Mosby, Agassiz trawl, 7 July $2001,65^{\circ} 3950 \mathrm{~N}, 9^{\circ} 0660 \mathrm{~W}, 818 \mathrm{~m},-0.58^{\circ} \mathrm{C}, 1$ specimen (IMNH 36961); BIOICE stn. 3198, RV Håkon

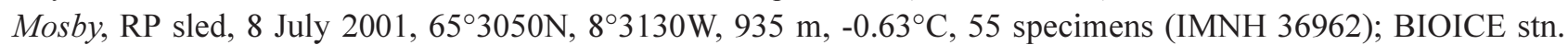
3199, RV Håkon Mosby, Agassiz trawl, 8 July 2001, 65 $3008 \mathrm{~N}, 8^{\circ} 3159 \mathrm{~W}, 934 \mathrm{~m},-0.63^{\circ} \mathrm{C}, 1$ specimen (IMNH 36963); BIOICE stn. 3204, RV Håkon Mosby, RP sled, 8 July 2001, 64ํำ $5171 \mathrm{~N}, 7^{\circ} 5456 \mathrm{~W}, 2613 \mathrm{~m},-0.83^{\circ} \mathrm{C}, 6$ specimens (IMNH 36964); BIOICE stn. 3219, RV Håkon Mosby, RP sled, 11 July 2001, 6701479N, $8^{\circ} 2806,1642$ m, $-0.82^{\circ} \mathrm{C}, 6$ specimens (IMNH 36965); BIOICE stn. 3225, RV Håkon Mosby, RP sled, 12 July 2001, 68 $3446 \mathrm{~N}$, $8^{\circ} 1555 \mathrm{~W}, 1993 \mathrm{~m},-0.86^{\circ} \mathrm{C}, 8$ specimens (IMNH 36966); BIOICE stn. 3280, RV Bjarni Scemundsson, RP sled, 16 September 2001, $62^{\circ} 5350 \mathrm{~N}, 15^{\circ} 5560 \mathrm{~W}, 1692 \mathrm{~m}, 2.85^{\circ} \mathrm{C}, 7$ specimens (IMNH 36967); BIOICE stn. 3501, RV Bjarni Scemundsson, RP sled, 31 August 2002, 62 $5984 \mathrm{~N}, 20^{\circ} 3025 \mathrm{~W}, 829 \mathrm{~m}, 5.82^{\circ} \mathrm{C}, 3$ specimens (IMNH 36968); BIOICE stn. 3515, RV Bjarni Scemundsson, RP sled, 4 September 2002, 62 $2220 \mathrm{~N}, 18^{\circ} 2335 \mathrm{~W}, 1331 \mathrm{~m}, 3.77^{\circ} \mathrm{C}$, 15 specimens (IMNH 36969); BIOICE stn. 3519, RV Bjarni Scemundsson, RP sled, 5 September 2002, 62 ${ }^{\circ} 1576$ N, $17^{\circ} 3256 \mathrm{~W}, 1960 \mathrm{~m}, 2.70^{\circ} \mathrm{C}, 1$ specimen (IMNH 36970); BIOICE stn. 3532, RV Bjarni Scemundsson, RP sled, 9 September 2002, $62^{\circ} 4310 \mathrm{~N}, 14^{\circ} 3291,1685 \mathrm{~m}, 2.51^{\circ} \mathrm{C}, 4$ specimens (IMNH 36971); BIOICE stn. 3534, RV Bjarni Scemundsson, Sneli sled, 9 September 2002, 62 ${ }^{\circ} 3819 \mathrm{~N}, 14^{\circ} 1446 \mathrm{~W}, 1556 \mathrm{~m}, 2.64^{\circ} \mathrm{C}, 1$ specimen (IMNH 36972); BIOICE stn. 3538, RV Bjarni Scemundsson, RP sled, 10 September $2002,62^{\circ} 2450 \mathrm{~N}, 14^{\circ} 1355 \mathrm{~W}, 1516 \mathrm{~m}, 2.57^{\circ} \mathrm{C}$, 9 specimens (IMNH 36973); BIOICE stn. 3539, RV Bjarni Scemundsson, RP sled, 10 September 2002, 61 ${ }^{\circ} 5963 \mathrm{~N}$, $13^{\circ} 3309 \mathrm{~W}, 1377 \mathrm{~m}, 2.41^{\circ} \mathrm{C}, 10$ specimens (IMNH 36974); BIOICE stn. 3543, RV Bjarni Scemundsson, RP sled, 11 September 2002, $61^{\circ} 3951 \mathrm{~N}, 13^{\circ} 5465 \mathrm{~W}, 1640 \mathrm{~m}, 2.86^{\circ} \mathrm{C}, 75$ specimens (IMNH 36975); BIOICE stn. 3592, RV Bjarni Scemundsson, RP sled, 9 September 2003, $61^{\circ} 4776 \mathrm{~N}, 27^{\circ} 0158 \mathrm{~W}, 888 \mathrm{~m}, 6.09^{\circ} \mathrm{C}, 4$ specimens (IMNH 36976); BIOICE stn. 3595, RV Bjarni Scemundsson, RP sled, 10 September 2003, 614958N, 260997W, 916 m, $5.52^{\circ} \mathrm{C}, 3$ specimens (IMNH 36977); BIOICE stn. 3598, RV Bjarni Scemundsson, RP sled, 10 September 2003, $62^{\circ} 1737 \mathrm{~N}, 25^{\circ} 3758 \mathrm{~W}, 769 \mathrm{~m}, 6.50^{\circ} \mathrm{C}, 3$ specimens (IMNH 36978); BIOICE stn. 3601, RV Bjarni Scemundsson, RP sled, 11 September $2003,62^{\circ} 4214 \mathrm{~N}, 25^{\circ} 2722 \mathrm{~W}, 624 \mathrm{~m}, 7.11^{\circ} \mathrm{C}, 2$ specimens (IMNH 36979); BIOICE stn. 3621, RV Håkon Mosby, RP sled, 14 July 2004, 66 ${ }^{\circ} 2939 \mathrm{~N}, 9^{\circ} 4480 \mathrm{~W}, 1475$ m, $-0.78{ }^{\circ} \mathrm{C}$, 3 specimens (IMNH 36980); BIOICE stn. 3624, RV Håkon Mosby, RP sled, 14 July 2004, 66 $5948 \mathrm{~N}, 8^{\circ} 4873,1628 \mathrm{~m},-0.82^{\circ} \mathrm{C}, 10$ specimens (IMNH 36981); BIOICE stn. 3627, RV Håkon Mosby, Agassiz trawl, 15 July 2004, 67¹973N, $9^{\circ} 3330 \mathrm{~W}, 1616 \mathrm{~m},-0.81^{\circ} \mathrm{C}, 2$ specimens (IMNH 36982); BIOICE stn. 3628, RV Håkon Mosby, RP sled, 15 July 2004, 67 $1953 \mathrm{~N}, 9^{\circ} 3276 \mathrm{~W}, 1609 \mathrm{~m},-0.81^{\circ} \mathrm{C}, 98$ specimens (IMNH 36983); BIOICE stn. 3629, RV Håkon Mosby, RP sled, 16 July $2004,67^{\circ} 5576 \mathrm{~N}, 8^{\circ} 1589,1481 \mathrm{~m},-0.84^{\circ} \mathrm{C}, 24$ specimens (IMNH 36984); BIOICE stn. 3632, RV Håkon Mosby, RP sled, 16 July 2004, $68^{\circ} 0092 \mathrm{~N}, 9^{\circ} 1478 \mathrm{~W}, 1727 \mathrm{~m},-0.82^{\circ} \mathrm{C}, 30$ specimens (IMNH 36985); BIOICE stn. 3633, RV Håkon Mosby, RP sled, 17 July $2004,68^{\circ} 2512 \mathrm{~N}, 8^{\circ} 5772 \mathrm{~W}, 1952 \mathrm{~m},-0.84^{\circ} \mathrm{C}, 35$ specimens (IMNH 36986); BIOICE stn. 3634, RV Håkon Mosby, Agassiz trawl, 17 July 2004, 68²552N, $8^{\circ} 5774 \mathrm{~W}, 1950 \mathrm{~m}$, $-0.84^{\circ} \mathrm{C}, 2$ specimens (IMNH 36987); BIOICE stn. 3636, RV Håkon Mosby, RP sled, 17 July 2004, 68 ${ }^{\circ} 4971 \mathrm{~N}$, $9^{\circ} 1442 \mathrm{~W}, 1844 \mathrm{~m},-0.81^{\circ} \mathrm{C}, 112$ specimens (IMNH 36988); BIOICE stn. 3637, RV Håkon Mosby, RP sled, 17 July 
2004, 68 $2692 \mathrm{~N}, 10^{\circ} 0875 \mathrm{~W}, 2069 \mathrm{~m},-0.80^{\circ} \mathrm{C}, 4$ specimens (IMNH 36989); BIOICE stn. 3638, RV Håkon Mosby, Agassiz trawl, 18 July 2004, 68 $2663 \mathrm{~N}, 10^{\circ} 1058 \mathrm{~W}, 2065 \mathrm{~m},-0.80^{\circ} \mathrm{C}, 52$ specimens (IMNH 36990 ); BIOICE stn. 3640, RV Håkon Mosby, RP sled, 18 July 2004, 67 $5763 \mathrm{~N}, 10^{\circ} 0261 \mathrm{~W}, 1915 \mathrm{~m},-0.82^{\circ} \mathrm{C}, 4$ specimens (IMNH 36991); BIOICE stn. 3641, RV Håkon Mosby, RP sled, 19 July 2004, 66 $5908 \mathrm{~N}, 10^{\circ} 4314 \mathrm{~W}, 1450 \mathrm{~m},-0.77^{\circ} \mathrm{C}, 15$ specimens (IMNH 36992); BIOICE stn. 3645, RV Håkon Mosby, RP sled, 21 July 2004, 67²422N, 104059W, $1703 \mathrm{~m},-0.81^{\circ} \mathrm{C}, 293$ specimens (IMNH 36993); BIOICE stn. 3648, RV Håkon Mosby, RP sled, 22 July 2004, $68^{\circ} 5710 \mathrm{~N}, 10^{\circ} 3280 \mathrm{~W}, 2215 \mathrm{~m},-0.79^{\circ} \mathrm{C}, 10$ specimens (IMNH 36994); BIOICE stn. 3649, RV Håkon Mosby, Agassiz trawl, 22 July 2004, 68 $5645 \mathrm{~N}, 10^{\circ} 3565 \mathrm{~W}, 2214 \mathrm{~m},-0.79^{\circ} \mathrm{C}, 6$ specimens (IMNH 36995); BIOICE stn. 3652, RV Håkon Mosby, RP sled, 22 July 2004, $69^{\circ} 0400 \mathrm{~N}, 13^{\circ} 3385 \mathrm{~W}, 1678 \mathrm{~m},-0.81^{\circ} \mathrm{C}, 65$ specimens (IMNH 36996); BIOICE stn. 3655, RV Håkon Mosby, RP sled, 23 July 2004, $68^{\circ} 4136 \mathrm{~N}, 14^{\circ} 1874 \mathrm{~W}, 1489 \mathrm{~m},-0.80^{\circ} \mathrm{C}, 127$ specimens (IMNH 36997); BIOICE stn. 3656, RV Håkon Mosby, RP sled, 23 July 2004, 68 $4711 \mathrm{~N}, 15^{\circ} 1867 \mathrm{~W}$, $1492 \mathrm{~m},-0.72^{\circ} \mathrm{C}, 23$ specimens (IMNH 36998); BIOICE stn. 3657, RV Håkon Mosby, Agassiz trawl, 23 July 2004, $68^{\circ} 4537 \mathrm{~N}, 15^{\circ} 2280 \mathrm{~W}, 1499 \mathrm{~m},-0.72^{\circ} \mathrm{C}, 1$ specimen (IMNH 36999); BIOICE stn. 3658, RV Håkon Mosby, RP sled, 24 July $2004,67^{\circ} 5978 \mathrm{~N}, 18^{\circ} 4838 \mathrm{~W}, 1018 \mathrm{~m},-0.60^{\circ} \mathrm{C}, 57$ specimens (IMNH 37000 ); BIOICE stn. 3659, RV Håkon Mosby, RP sled, 24 July 2004, $67^{\circ} 4761 \mathrm{~N}, 19^{\circ} 3638 \mathrm{~W}, 806 \mathrm{~m},-0.53^{\circ} \mathrm{C}, 104$ specimens (IMNH 37001 ).

Remarks. This species is very common in the region, reported from 136 stations with most of them (100 stations) north of the Greenland-Iceland-Faeroe Ridge (Fig. 11A). The distribution of the species on the GIF Ridge is, however, peculiar as the species is absent from shallow waters south of the Ridge, being found there at depths between 624 and $2082 \mathrm{~m}$ (Table 1). North of the Ridge the species occurs at depths between 64 and $2613 \mathrm{~m}$, and with all but one record at $294 \mathrm{~m}$ and deeper. The distribution is though continuous as the bathymetrical distribution of the species on both sides of the Ridge reaches above the deepest saddle depth of the Ridge, i.e. $840 \mathrm{~m}$ (Hansen \& Østerhus 2000). North of Iceland, H. bicuspis was generally found at low temperatures (in all cases but one at $<1.68^{\circ} \mathrm{C}$, one locality at $5.17^{\circ} \mathrm{C}$ ) and mostly at temperatures below zero. South of the Ridge the temperatures ranged from 2.4 to $7.11^{\circ} \mathrm{C}$.

TABLE 1. List of haploniscid species occurring in the GIF area, the number of stations, number of individuals, minimum and maximum depths area (north or south of the Ridge), minimum and maximum temperature and water mass (es).

\begin{tabular}{|c|c|c|c|c|c|c|c|c|}
\hline Species & $\begin{array}{l}\text { No. of } \\
\text { stations }\end{array}$ & $\begin{array}{l}\text { No. of } \\
\text { individuals }\end{array}$ & $\begin{array}{l}\text { Min. } \\
\text { depth } \\
\text { (m) }\end{array}$ & $\begin{array}{l}\text { Max } \\
\text { depth } \\
\text { (m) }\end{array}$ & Area & $\begin{array}{l}\text { Min. } \\
\text { temp. } \\
\left({ }^{\circ} \mathrm{C}\right)\end{array}$ & $\begin{array}{l}\text { Max. } \\
\text { temp. } \\
\left({ }^{\circ} \mathrm{C}\right)\end{array}$ & Water mass(es) \\
\hline Haploniscus astraphes & 1 & 2 & 1300 & & $\mathrm{~S}$ & 3.96 & & LSW \\
\hline Haploniscus aduncus & 10 & 31 & 1543 & 2537 & $\mathrm{~S}$ & 2.37 & 3.7 & ISOW, LSW \\
\hline Haploniscus ampliatus & 1 & 4 & 2359 & & $\mathrm{~S}$ & 3.06 & & LSW \\
\hline Haploniscus angustus & 4 & 7 & 794 & 2271 & $\mathrm{~N}$ and $\mathrm{S}$ & -0.53 & 2.37 & $\begin{array}{l}\text { ISOW, probably } \\
\text { NSAIW }\end{array}$ \\
\hline Haploniscus bicuspis & 136 & 11410 & 64 & 2613 & $\mathrm{~N}$ and $\mathrm{S}$ & -0.86 & 7.11 & $\begin{array}{l}\text { MNAW, LSW, } \\
\text { ISOW, NSAIW, } \\
\text { NSDW }\end{array}$ \\
\hline Haploniscus foresti & 19 & 1375 & 1295 & 2400 & $\mathrm{~S}$ & 2.07 & 3.99 & ISOW, LSW \\
\hline Haploniscus hamatus & 1 & 1 & 2270 & & $\mathrm{~S}$ & 2.37 & & ISOW \\
\hline Haploniscus spinifer & 2 & 96 & 2270 & 2295 & $\mathrm{~S}$ & 2.37 & 2.6 & ISOW \\
\hline Antennuloniscus simplex & 12 & 1816 & 1558 & 2295 & $\mathrm{~S}$ & 2,34 & 3,77 & ISOW, LSW \\
\hline $\begin{array}{l}\text { Chauliodoniscus } \\
\text { armadilloides }\end{array}$ & 7 & 19 & 1516 & 2537 & $\mathrm{~S}$ & 2.37 & 3.29 & ISOW, LSW \\
\hline
\end{tabular}

Haploniscus bicuspis has a very wide distribution, occurring both in the North and the South Atlantic (Brökeland \& Wägele 2004). The record by Menzies (1962) of H. bicuspis from the South Atlantic probably refers to another species (Brökeland \& Wägele 2004), since the illustration by Menzies (1962) shows a specimen differing from H. bicuspis in important characters, such as the shape of the rostrum. 


\section{Haploniscus foresti Chardy, 1974}

Haploniscus foresti Chardy, 1974: 497-498, figs 1-2.—Lincoln 1985b: 669-673, figs 7-8.—Kussakin 1988: 399-402, fig. 338.- Kavanagh 2009: 30.

Material examined. BIOICE stn. 2704, RV Bjarni Scemundsson, RP sled, 20 September 1994, 635050N, $27^{\circ} 4280 \mathrm{~W}, 1295 \mathrm{~m}, 3.80^{\circ} \mathrm{C}, 10$ specimens (IMNH 37002); BIOICE stn. 2856, RV Bjarni Scemundsson, RP sled, 29 August 1995, 62 $2050 \mathrm{~N}, 16^{\circ} 5930 \mathrm{~W}, 2074 \mathrm{~m}, 2.34^{\circ} \mathrm{C}, 1$ specimen (IMNH 37003); BIOICE stn. 2859, RV Bjarni Scemundsson, RP sled, 29 August 1995, 61 ${ }^{\circ} 5022 \mathrm{~N}, 16^{\circ} 5286 \mathrm{~W}, 2270 \mathrm{~m}, 2.37^{\circ} \mathrm{C}, 36$ specimens (IMNH 37004); BIOICE stn. 2860, RV Bjarni Scemundsson, RP sled, 30 August 1995, 614343N, 165748W, 2295 m, $2.60^{\circ} \mathrm{C}, 55$ specimens (IMNH 37005); BIOICE stn. 2863, RV Bjarni Scemundsson, RP sled, 30 August 1995, 61¹018N, $18^{\circ} 0257 \mathrm{~W}, 2400 \mathrm{~m}, 2.07^{\circ} \mathrm{C}$, 19 specimens (IMNH 37006); BIOICE stn. 3012, RV Bjarni Scemundsson, RP sled, 7 July $1997,61^{\circ} 2220 \mathrm{~N}, 15^{\circ} 1898 \mathrm{~W}, 2133 \mathrm{~m}, 3.29^{\circ} \mathrm{C}, 7$ specimens (IMNH 37007); BIOICE stn. 3075, RV Bjarni

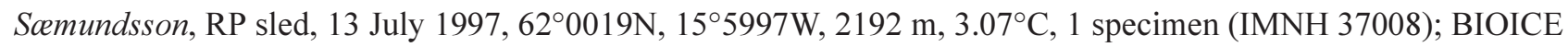
stn. 3164, RV Bjarni Scemundsson, RP sled, 26 July 2000, $61^{\circ} 4260 \mathrm{~N}, 22^{\circ} 5750 \mathrm{~W}, 1741 \mathrm{~m}, 2.79^{\circ} \mathrm{C}, 20$ specimens (IMNH 37009); BIOICE stn. 3167, RV Bjarni Scemundsson, RP sled, 26 July 2000, 605488N, 22॰4726W, 1897 m, $2.98^{\circ} \mathrm{C}$, 19 specimens (IMNH 37010); BIOICE stn. 3187, RV Bjarni Scemundsson, RP sled, 10 August 2000 , $62^{\circ} 0904 \mathrm{~N}, 27^{\circ} 0074 \mathrm{~W}, 1327 \mathrm{~m}, 3.99^{\circ} \mathrm{C}, 2$ specimens (IMNH 37011 ); BIOICE stn. 3264, RV Bjarni Scemundsson, RP sled, 13 September 2001, 62 ${ }^{\circ} 2480 \mathrm{~N}, 19^{\circ} 4860 \mathrm{~W}, 1780 \mathrm{~m}, 2.95^{\circ} \mathrm{C}, 1$ specimen (IMNH 37012 ); BIOICE stn. 3504, RV Bjarni Scemundsson, RP sled, 20 September 2002, 62 $0146 \mathrm{~N}, 19^{\circ} 4915 \mathrm{~W}, 1733 \mathrm{~m}, 3.09^{\circ} \mathrm{C}, 28 \mathrm{specimens}$ (IMNH 37013); BIOICE stn. 3509, RV Bjarni Scemundsson, RP sled, 30 September 2002, 620240N, 19³871 W, 1678 m, 2.70 C, 203 specimens (IMNH 37014); BIOICE stn. 3514, RV Bjarni Scemundsson, RP sled, 4 September 2002, 62 $2559 \mathrm{~N}, 19^{\circ} 4615 \mathrm{~W}, 1780 \mathrm{~m}, 2.93^{\circ} \mathrm{C}, 893$ specimens (IMNH 37015); BIOICE stn. 3515, RV Bjarni Scemundsson, RP sled, 4 September 2002, 62 $2220 \mathrm{~N}, 18^{\circ} 2335 \mathrm{~W}, 1331 \mathrm{~m}, 3.77^{\circ} \mathrm{C}, 3$ specimens (IMNH 37016); BIOICE stn. 3522, RV Bjarni Scemundsson, RP sled, 7 September 2002, 62 $3114 \mathrm{~N}, 17^{\circ} 0987 \mathrm{~W}, 1940 \mathrm{~m}, 2.34^{\circ} \mathrm{C}, 7$ specimens (IMNH 37017); BIOICE stn. 3570, RV Bjarni Scemundsson, RP sled, 4 September 2003, 634271N, $29^{\circ} 1036 \mathrm{~W}, 1819 \mathrm{~m}, 3.24^{\circ} \mathrm{C}, 28$ specimens (IMNH 37018); BIOICE stn. 3571, RV Bjarni Scemundsson, RP sled, 5 September 2003, 633022N, 293840W, 2233 m, 3.14 ${ }^{\circ} \mathrm{C}$, 1 specimen (IMNH 37019); BIOICE stn. 3579, RV Bjarni Scemundsson, RP sled, 6 September 2003, 630.442N, 28 $2639 \mathrm{~W}, 1796$ m, 3.42 ${ }^{\circ} \mathrm{C}, 41$ specimens (IMNH 37020).

Remarks. New records of Haploniscus foresti include 19 samples collected in the Iceland Basin and the Irminger Basin at depths between 1295 and 2400 m (Fig. 11B, Table 1). Previous records of H. foresti are from the Rockall Trough, the Rockall Plateau (type locality, Chardy 1974) and the Porcupine Seabight and at the depth range of 1632 and $3697 \mathrm{~m}$ (Lincoln 1985b; Kavanagh 2009). The BIOICE samples extend the known range of the species to nearly $64^{\circ} \mathrm{N}$ and $29^{\circ} \mathrm{W}$.

\section{Haploniscus hamatus Lincoln, 1985}

Haploniscus hamatus Lincoln, 1985b: 666-669, figs 5-6.-Kussakin 1988: 381-383, fig. 323.—Kavanagh $2009: 30$.

Material examined. BIOICE stn. 2859, RV Bjarni Scemundsson, RP sled, 29 August 1995, 61 ${ }^{\circ} 5022 \mathrm{~N}, 16^{\circ} 5286 \mathrm{~W}$, $2270 \mathrm{~m}, 2.37^{\circ} \mathrm{C}, 1$ specimen (IMNH 37021 ).

Remarks. Only a single specimen was collected of H. hamatus, being found in the Iceland Basin at depth of $2270 \mathrm{~m}$ (Fig. 10A, square). Previous reports of the species are from the Rockall Trough and the Wyville-Thompson Ridge at depths from 900 to 2925 m (Lincoln 1985b; Kavanagh 2009).

\section{Haploniscus spinifer Hansen, 1916}

Haploniscus spinifer Hansen, 1916: 31, pl. II, figs 3a-3h._-Menzies 1962: 98.—Wolff 1962: 59.-Chardy 1974: 496, 523.— Lincoln 1985b: 662-663.-Kussakin 1988: 396-397, figs 333-334.-Brökeland and Wägele 2004: 237-238, fig. 2 (Electronic supplement, 4-6, figs 13-25). 


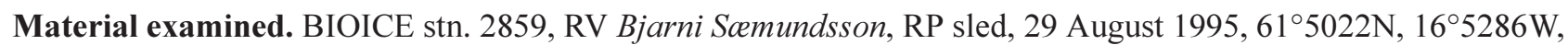
$2270 \mathrm{~m}, 2.37^{\circ} \mathrm{C}, 39$ specimens (IMNH 37022); BIOICE stn. 2860, RV Bjarni Scemundsson, RP sled, 30 August $1995,61^{\circ} 4343 \mathrm{~N}, 16^{\circ} 5748 \mathrm{~W}, 2295 \mathrm{~m}, 2.60^{\circ} \mathrm{C}, 57$ specimens (IMNH 37023 ).

Remarks. Hansen (1916) described H. spinifer from specimens collected at depths of 1505-3474 $\mathrm{m}$ in the Labrador basin and at the eastern margin of the deep Reykjanes Ridge. Here, the species was only found at two deep stations south of the Ridge in the Iceland basin (Fig. 11C). Lincoln (1985b) did not report the species from the Rockall Trough or the Porcupine Sea-Bight. The species has a very wide distribution and has been reported in the South Atlantic Ocean (Brökeland \& Wägele 2004) and in even in the Southern Ocean (see Meyer-Löbbecke et al. 2014), and has been suggested to be a potential species complex.

\section{Antennuloniscus simplex Lincoln, 1985}

Antennuloniscus simplex Lincoln, 1985b: 687-690, figs 18-19.—Kussakin 1988: 412-414, fig. 344.-Kavanagh $2009: 27$.

Material examined. BIOICE stn. 2853, RV Bjarni Scemundsson, RP sled, 28 August 1995, 62 ${ }^{\circ} 4030 \mathrm{~N}, 16^{\circ} 5820 \mathrm{~W}$, $1833 \mathrm{~m}, 2.40^{\circ} \mathrm{C}, 877$ specimens (IMNH 36832); BIOICE stn. 2854, RV Bjarni Scemundsson, RP sled, 28 August 1995, 62 $2017 \mathrm{~N}, 16^{\circ} 5940 \mathrm{~W}, 2074 \mathrm{~m}, 2.34^{\circ} \mathrm{C}, 1$ specimen (IMNH 36833); BIOICE stn. 2860, RV Bjarni Scemundsson, RP sled, 30 August 1995, 61 ${ }^{\circ} 4343 \mathrm{~N}, 16^{\circ} 5748 \mathrm{~W}, 2295 \mathrm{~m}, 2.60^{\circ} \mathrm{C}, 2$ specimens (IMNH 36834); BIOICE stn. 3069, RV Bjarni Scemundsson, RP sled, 11 July 1997, 62 ${ }^{\circ} 2880 \mathrm{~N}, 14^{\circ} 2994 \mathrm{~W}, 1602 \mathrm{~m}, 3.24^{\circ} \mathrm{C}, 419$ specimens (IMNH 36835); BIOICE stn. 3070, RV Bjarni Scemundsson, Agassiz trawl, 12 July 1997, 62 ${ }^{\circ} 1686 \mathrm{~N}$, $15^{\circ} 2016 \mathrm{~W}, 2069 \mathrm{~m}, 3.19^{\circ} \mathrm{C}, 296$ specimens (IMNH 36836); BIOICE stn. 3072, RV Bjarni Scemundsson, RP sled, 12 July $1997,61^{\circ} 5365 \mathrm{~N}, 15^{\circ} 0815 \mathrm{~W}, 2082 \mathrm{~m}, 2.83^{\circ} \mathrm{C}, 171$ specimens (IMNH 36837); BIOICE stn. 3074, RV Bjarni Scemundsson, Sneli sled, 12 July 1997, 61 ${ }^{\circ} 5427 \mathrm{~N}, 15^{\circ} 0877,2085 \mathrm{~m}, 2.83^{\circ} \mathrm{C}, 3$ specimens (IMNH 36838); BIOICE stn. 3519, RV Bjarni Scemundsson, RP sled, 5 September 2002, 62 ${ }^{\circ} 1576 \mathrm{~N}, 17^{\circ} 3256 \mathrm{~W}, 1960 \mathrm{~m}, 2.70^{\circ} \mathrm{C}, 5$ specimens (IMNH 36839); BIOICE stn. 3522, RV Bjarni Scemundsson, RP sled, 7 September 2002, 62 ${ }^{\circ} 3114 \mathrm{~N}$, $17^{\circ} 0987 \mathrm{~W}, 1940 \mathrm{~m}, 2.34^{\circ} \mathrm{C}, 1$ specimen (IMNH 36840); BIOICE stn. 3532, RV Bjarni Scemundsson, RP sled, 9 September 2002, $62^{\circ} 4310 \mathrm{~N}, 14^{\circ} 3291,1685 \mathrm{~m}, 2.51^{\circ} \mathrm{C}, 24$ specimens (IMNH 36841); BIOICE stn. 3576, RV Bjarni Scemundsson, RP sled, 6 September 2003, 63 ${ }^{\circ} 1746$ N, $28^{\circ} 3439 \mathrm{~W}, 1744 \mathrm{~m}, 3.70^{\circ} \mathrm{C}, 16$ specimens (IMNH 36842); BIOICE stn. 3586, RV Bjarni Scemundsson, RP sled, 7 September 2003, 62 $2315 \mathrm{~N}, 28^{\circ} 1691 \mathrm{~W}, 1558 \mathrm{~m}$, $3.77^{\circ} \mathrm{C}, 1$ specimen (IMNH 36843 ).

Remarks. Previously Antennuloniscus simplex was known only from the type locality in the Rockall Trough at $1900 \mathrm{~m}$ (Lincoln 1985b). During the BIOICE project it was sampled mainly at several neighbouring stations in the Iceland basin and at two station in the Irmiger Basin, south of the Iceland-Faeroe Ridge at depths between 1558 and $2295 \mathrm{~m}$ (Fig. 12A, Table 1), extending the known distribution of the species to about $63^{\circ} \mathrm{N}$.

\section{Chauliodoniscus armadilloides (Hansen, 1916)}

Haploniscus armadilloides Hansen, 1916: 31-32; pl. 2, fig. 4a-d.-Gurjanova 1933: 402.—Wolff 1962: 55, 217, 259, 274.Chardy 1974: 496, 523.

Chauliodoniscus armadilloides —Lincoln 1985a: 35.-Lincoln 1985b: 682-687, figs 16-17.-Kussakin 1988: 402-404, fig. 339.--Kavanagh 2009: 28.

Material examined. BIOICE stn. 2853, RV Bjarni Scemundsson, RP sled, 28 August 1995, 62 ${ }^{\circ} 4030 \mathrm{~N}, 16^{\circ} 5820 \mathrm{~W}$, $1833 \mathrm{~m}, 2.40^{\circ} \mathrm{C}, 1$ specimen (IMNH 36844); BIOICE stn. 2859, RV Bjarni Scemundsson, RP sled, 29 August 1995, $61^{\circ} 5022 \mathrm{~N}, 16^{\circ} 5286 \mathrm{~W}, 2270 \mathrm{~m}, 2.37^{\circ} \mathrm{C}, 5$ specimens (IMNH 36845); BIOICE stn. 2860, RV Bjarni Scemundsson, RP sled, 30 August 1995, 61 ${ }^{\circ} 4343 \mathrm{~N}, 16^{\circ} 5748 \mathrm{~W}, 2295 \mathrm{~m}, 2.60^{\circ} \mathrm{C}, 3$ specimens (IMNH 36846); BIOICE stn. 3012, RV Bjarni Scemundsson, RP sled, 7 July 1997, 61 ${ }^{\circ} 220 \mathrm{~N}, 15^{\circ} 1898 \mathrm{~W}, 2133 \mathrm{~m}, 3.29^{\circ} \mathrm{C}, 5$ specimens (IMNH 36847); BIOICE stn. 3176, RV Bjarni Scemundsson, RP sled, 29 July 2000, 600203N, $22^{\circ} 2717 \mathrm{~W}, 2537 \mathrm{~m}, 3$ specimens (IMNH 36848); BIOICE stn. 3280, RV Bjarni Scemundsson, RP sled, 16 September 2001, 62 ${ }^{\circ} 5350 \mathrm{~N}$, $15^{\circ} 5560 \mathrm{~W}, 1692 \mathrm{~m}, 2.85^{\circ} \mathrm{C}, 1$ specimen (IMNH 36849); BIOICE stn. 3538, RV Bjarni Scemundsson, RP sled, 10 September $2002,62^{\circ} 2450 \mathrm{~N}, 14^{\circ} 1355 \mathrm{~W}, 1516 \mathrm{~m}, 2.57^{\circ} \mathrm{C}, 1$ specimen (IMNH 36850 ). 
Remarks. Chauliodoniscus armadilloides was found at seven stations. Six of these stations were located near the type locality $\left(63^{\circ} 08^{\prime} \mathrm{N}, 15^{\circ} 40^{\prime} \mathrm{W}, 1301 \mathrm{~m}\right.$, Hansen 1916$)$ in the Iceland basin south of the Iceland-Faeroe Ridge, and the seventh locality is in the southwestern part of the Iceland basin, somewhat deeper $(2537 \mathrm{~m})$ than the other stations (Fig. 12B). Other records are from the Rockall Trough and the Porcupine Seabight in depth down to $3550 \mathrm{~m}$ (Lincoln 1985b) and from the North Atlantic at $36^{\circ}$ to $45^{\circ} \mathrm{N}$, at depths between 3663 and $5240 \mathrm{~m}$ (Chardy 1974). Harrison (1989) found this species to be the second most common species at the Permanent station in the southern Rockall Trough.

\section{Distribution of Haploniscidae at the Greenland-Iceland-Faeroe Ridge}

Ten species of the Haploniscidae were sampled during the BIOICE expeditions in the GIF area (Table 1, Figs 1012), eight species of Haploniscus and one species each of genera Antennuloniscus and Chauliodoniscus. By far the most frequent species was Haploniscus bicuspis with 11,410 specimens sampled at 136 stations (Table 1). $H$. foresti and A. simplex were also fairly frequent, occurring at 19 and 12 stations, with 1375 and 1816 specimens, respectively. Haploniscus aduncus occurred at ten stations, while Chauliodoniscus armadilloides was reported from 7 stations. The remaining species were found at one to four stations only, i.e. Haploniscus astraphes, $H$. ampliatus, H. angustus, H. hamatus, and H. spinifer.

The only species occurring north of the Greenland-Iceland-Faeroe Ridge were Haploniscus bicuspis and $H$. angustus. Haploniscus ingolfi is the only other haploniscid species previously reported from the Nordic Seas (Wolff 1962; Svavarsson 1988), but was not found is this study.

H. bicuspis and H. angustus were the only haploniscid species occurring at depths shallower than the saddle depth at $840 \mathrm{~m}$ on the Ridge and seem to be able to cross the shallow sills of the Greenland-Iceland-Faeroe Ridge and thus colonize the waters all around Iceland. Haploniscus bicuspis has been reported to be adapted to cold conditions around $0^{\circ} \mathrm{C}$ (Hansen 1916; Wolff 1962), and was here reported in many instances from temperatures below zero (as low as $-0.86^{\circ} \mathrm{C}$ ), but even at temperatures as high as $7.11^{\circ} \mathrm{C}$. Accordingly, the species was found in a variety of water masses, such as the warm Modified North Atlantic Water (MNAW), the deep Labrador Sea Water (LSW) and the Iceland Sea Overflow Water (ISOW) south of Iceland, and even in the cold Norwegian Sea Deep Water (NSDW) in the deep north.

The remaining species are confined to areas south of the Greenland-Iceland-Faeroe Ridge (Figs 10-12) and also to depths greater than $1200 \mathrm{~m}$, holding higher $\left(>2^{\circ} \mathrm{C}\right)$ bottom temperatures (Table 1). H. astraphes and $H$. ampliatus occurred only in the LSW, while H. hamatus and H. spinifer occurred only in the ISOW. Haploniscus aduncus, H. foresti, Antennuloniscus simplex and Chauliodoniscus armadilloides occurred, however, in both ISOW and LSW.

The Greenland-Iceland-Faeroe Ridge is presumably one of the most pronounced barriers of species distribution in the world oceans. This is the only extensive, shallow ridge crossing the Atlantic Ocean in an easterly-westerly direction, separating the Nordic Seas and the Arctic Ocean from the North Atlantic proper. The deepest connections between these oceans are the Denmark Strait $(\sim 620 \mathrm{~m})$ in the Greenland-Iceland Ridge and the Faroe Bank Channel $(\sim 840 \mathrm{~m})$ southwest of the Faeroe Islands (Hansen \& Østerhus 2000$)$. North of the ridge bottom temperatures are low, being nearly $-0.9^{\circ} \mathrm{C}$ in the deepest parts. The cold overflow water passing the ridge mixes with warmer water masses resulting in higher bottom temperatures south of the ridge.

Most commonly benthic species living in the area have their distributional limits at the ridge, e.g. desmosomatid and nannoniscoid asellote isopods (Brix \& Svavarsson 2010); anthuridean isopods (Negoescu \& Svavarsson 1997), valviferan isopod (Stransky \& Svavarsson 2006), amphipods (Weisshappel 2001) and pectinoid bivalve molluscs (Dijkstra et al. 2009). It is still poorly known how the ridge and the different associated water masses shape the distribution of the species in question; whether this is by the strong thermal gradient, by the presence of water masses of subzero temperatures being difficult to adapt to, or simply by the physical presence of the ridge itself. Svavarsson et al. (1993) concluded that the diversity of the Arctic deep-water fauna was low due to the isolating effects of the ridge and the associated water masses.

Although haploniscid isopods are known throughout the world oceans, the Nordic Seas and the Arctic Ocean are poor of haploniscid species (Svavarsson et al. 1993). Apparently, the physical presence of the ridge limits the distribution of most of the haploniscid species towards the north, due to the deep occurrence of most of the species seen here. 

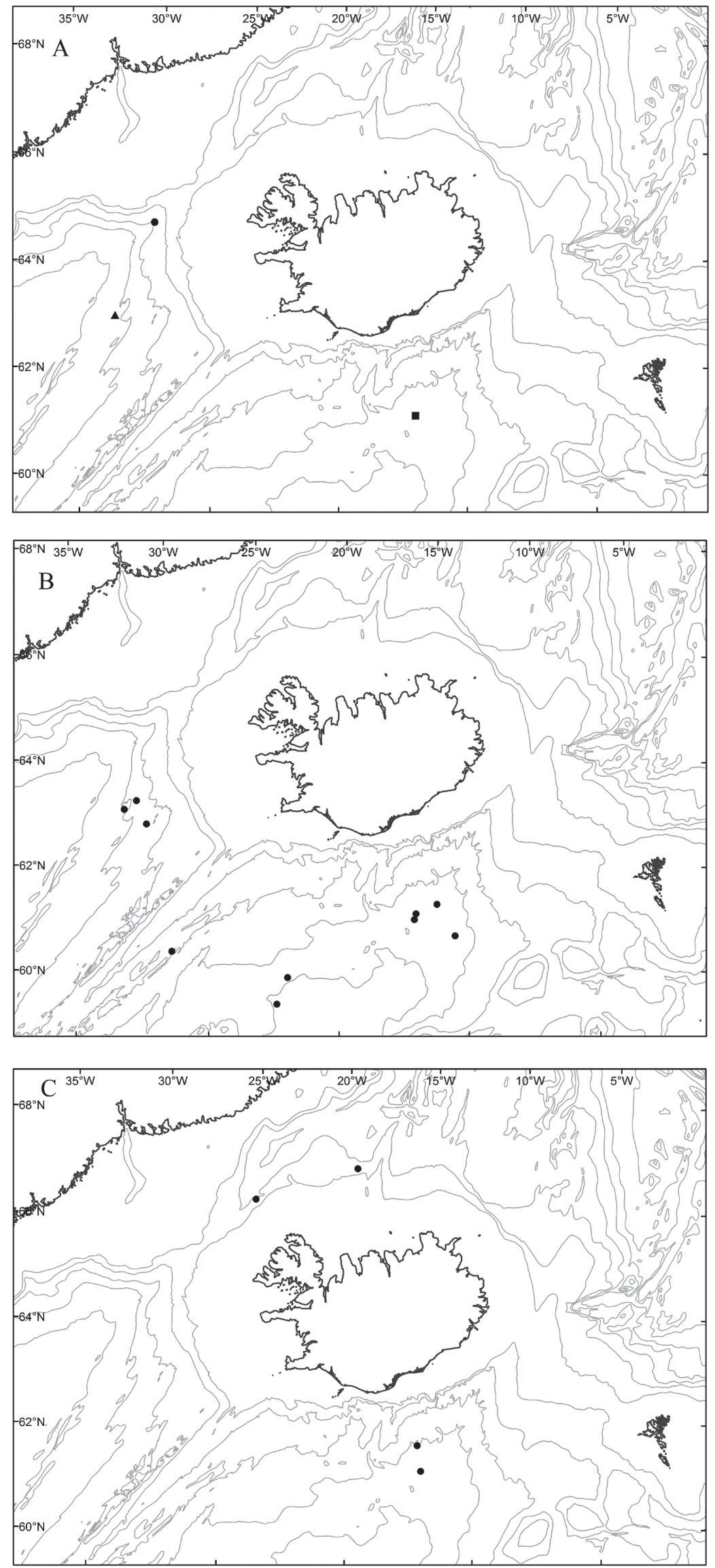

FIGURE 10. Distribution of haploniscid species around Iceland (BIOICE localities): A, Haploniscus astraphes n. sp. (dot), H. ampliatus (triangle) and H. hamatus (square); B, H. aduncus; C, H. angustus. 

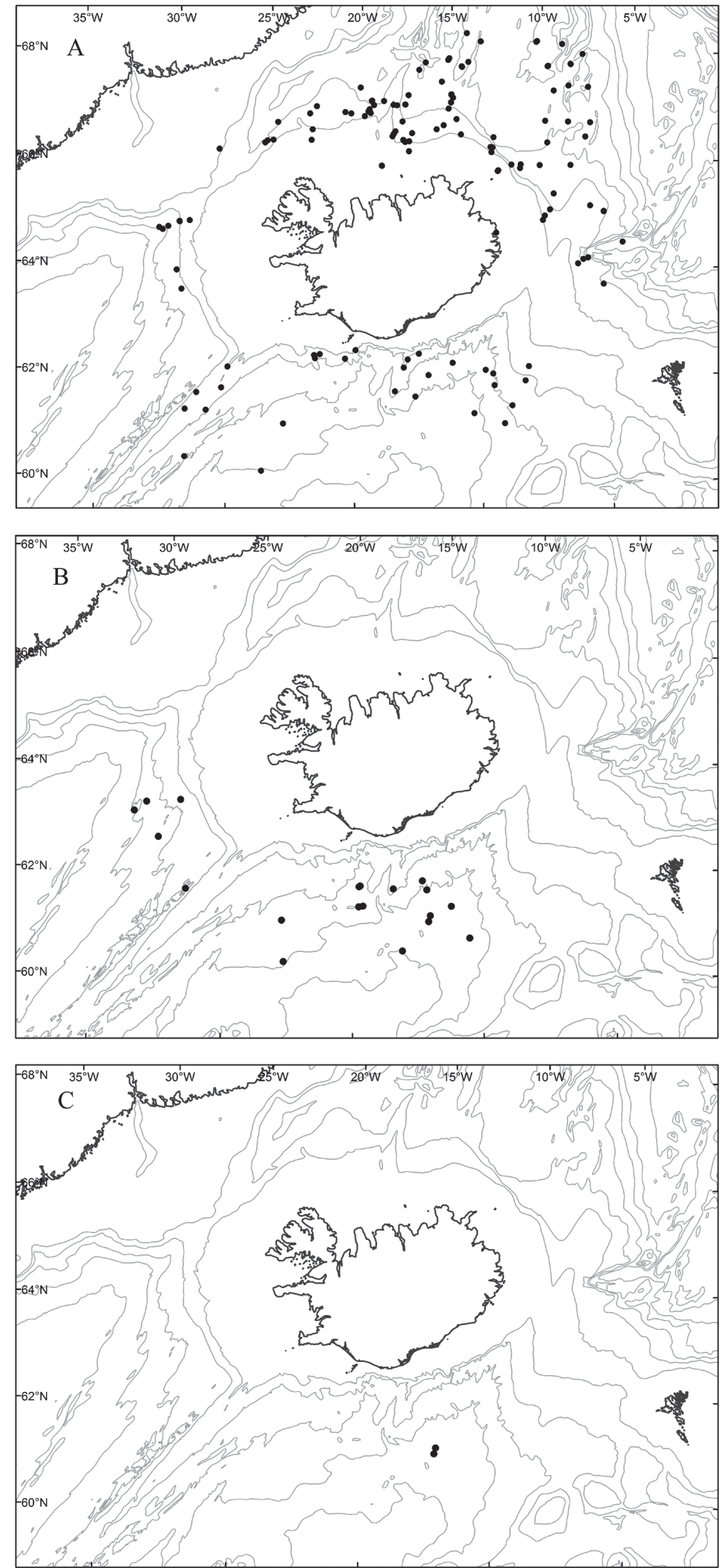

FIGURE 11. Distribution of haploniscid species around Iceland (BIOICE localities): A, H. bicuspis; B, H. foresti; C, H. spinifer. 

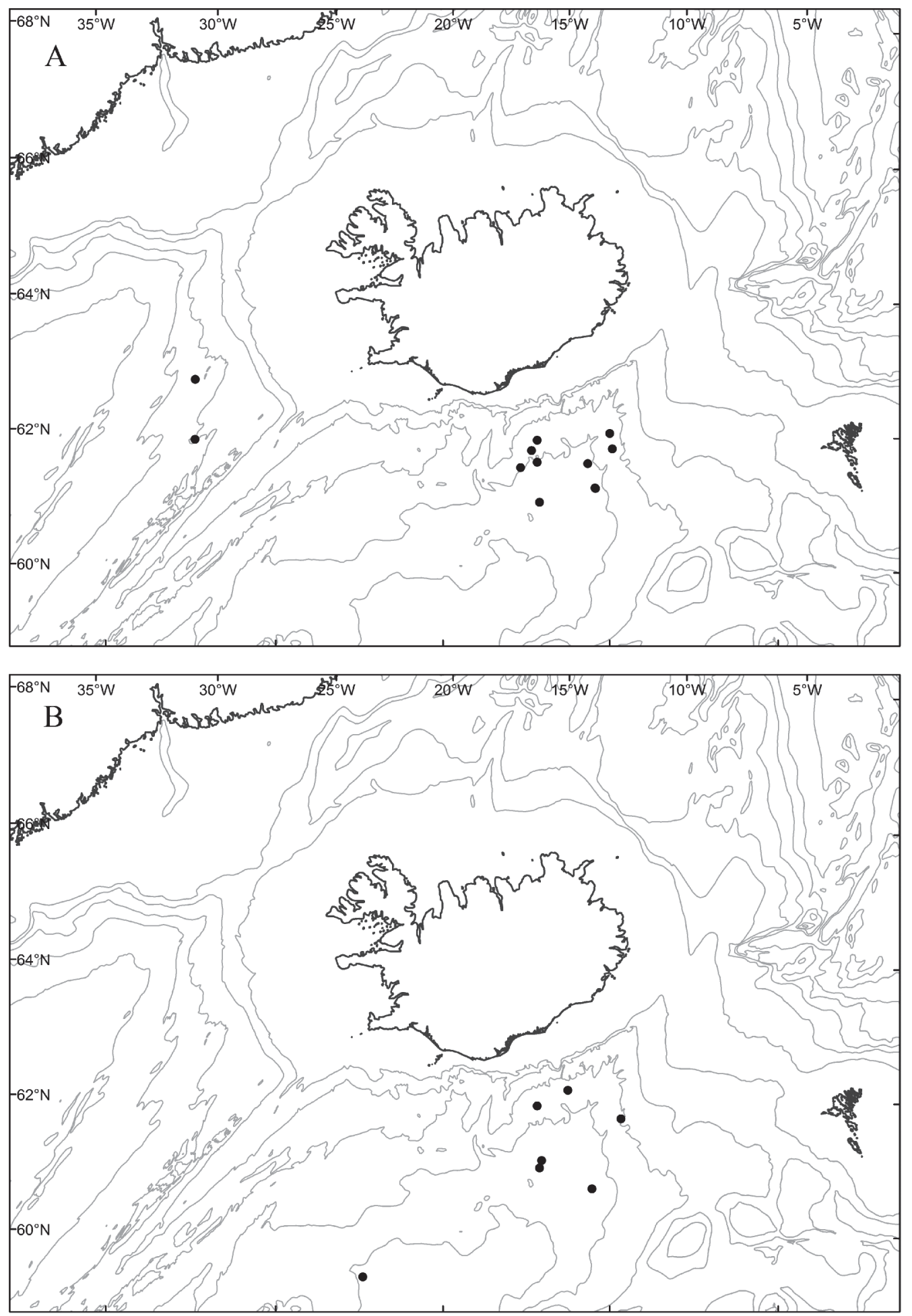

FIGURE 12. Distribution of haploniscid species around Iceland (BIOICE localities): A, Antennuloniscus simplex; B, Chauliodoniscus armadilloides.

Most of the haploniscids listed here have been found in the northernmost part of the Atlantic Ocean. Haploniscus astraphes n. sp., however, shows an extensive distribution, from the south side of the GreenlandIceland Ridge to the Guinea Basin, South Atlantic and the Angola Basin. Whether these really belong to the same species remains to be evaluated using molecular methods. 


\section{Acknowledgments}

We wish to thank Torleiv Brattegard for his hospitality onboard the RV Håkon Mosby, MRI, Reykjavík, for the use of RV Bjarni Scemundsson and the Fiskirannsóknastofa, Torshavn, for the use of RV Magnus Heinason. We would like to thank Sigrún Haraldsdóttir and the staff at the Sandgerði Marine Centre for sorting the BIOICE isopods, Nils Brenke for providing the DIVA specimens and Rósa Ólafsdóttir for making the maps. We thank two anonymous reviewers and Kenneth Meland for their constructive comments. We acknowledge a grant from the University of Iceland Research Fund.

\section{References}

Ahyong, S.T., Lowry, J.K., Alonso, M., Bamber, R.N., Boxshall, G.A., Castro, P., Gerken, S., Karaman, G.S., Goy, J.W., Jones, D.S., Meland, K., Rogers, D.C. \& Svavarsson, J. (2011) Subphylum Crustacea Brünnich, 1772. In: Zhang, Z.-Q. (Ed.), Animal biodiversity: An outline of higher-level classification and survey of taxonomic richness. Zootaxa, 3148, $165-191$.

Birstein, J.A. (1969) Crustacea Isopoda from the Romanche Trench. Bulletin of Moscow Society of Naturalists, Biological Series, 74 (3), 50-59.

Boyko., C.B., Bruce, N.L., Merrin, K.L., Ota, Y., Poore, G.C.B., Taiti, S., Schotte, M. \& Wilson, G.D.F. (Eds.) (2008 onwards). World list of marine freshwater and terrestrial isopod crustaceans. Available from: http://www.marinespecies.org/isopoda (accessed 22 November 2016)

Brattegard, T. \& Fosså, J.H. (1991) Replicability of an epibenthic sampler. Journal of the Marine Biological Association of the United Kingdom, 71, 153-166. https://doi.org/10.1017/S0025315400037462

Brenke, N. (2005) An epibenthic sledge for operations on marine soft bottom and bedrock. Marine Technology Society Journal, 39 (2), 10-21. https://doi.org/10.4031/002533205787444015

Brix, S. \& Svavarsson, J. (2010) Distribution and diversity of desmosomatid and nannoniscid isopods (Crustacea) on the Greenland-Iceland-Faeroe Ridge. Polar Biology, 33, 525-530. https://doi.org/10.1007/s00300-009-0729-8

Brökeland, W. (2006) Three species of the isopod crustacean genus Antennuloniscus Menzies, 1962 (Asellota: Haploniscidae) from the Southern Ocean. Zootaxa, 1115, 1-29.

Brökeland, W. (2010) Redescription of Haploniscus rostratus (Menzies, 1962) (Crustacea: Peracarida: Isopoda) with observations on the postmarsupial development, size ranges and distribution. Zootaxa, 2521, 1-25.

Brökeland, W. \& Wägele, J.-W. (2004) Redescription of three species of Haploniscus Richardson, 1908 (Isopoda, Asellota, Haploniscidae) from the Angola Basin. Organisms Diversity \& Evolution, 4, 237-239. [Electronic Supplement, 7, 1-40]

Chardy, P. (1974) Les Haploniscidae (Crustacés Isopodes Asellotes) de l'Atlantique. Description de huit espèces nouvelles. Bulletin du Musèum National d'Histoire Naturelle (3) Zoologie, 167 (243), 1137-1166.

Dijkstra, H.H., Warén, A. \& Guðmundsson, G. (2009) Pectinoidea (Mollusca: Bivalvia) from Iceland. Marine Biology Research, 5, 207-243. https://doi.org/10.1080/17451000802425643

Gurjanova, E.F. (1933) Die marinen Isopoden der Arktis. Fauna Arctica, 6 (5), 391-470.

Hansen, H.J. (1916) Crustacea Malacostraca. The order Isopoda. The Danish Ingolf Expedition, 3 (5), 1-262.

Hansen, B. \& Østerhus, S. (2000) North Atlantic-Nordic Seas exchanges. Progress in Oceanography, 45, 109-208. https://doi.org/10.1016/S0079-6611(99)00052-X

Harrison, K. (1989) Are deep-sea asellote isopods infaunal or epifaunal. Crustaceana, 56, 317-319. https://doi.org/10.1163/156854089X00284

Kavanagh, F.A. (2009) A catalogue of the Asellota (Crustacea: Isopoda) off the west coast of Ireland and Britain, from 100$5000 \mathrm{~m}$. Bulletin of the Irish biogeographical Society, 33, 14-75.

Lincoln, R.J. (1985a) The marine Fauna of New Zealand: Deep-sea Isopoda Asellota, family Haploniscidae. Memoirs of the New Zealand Oceanographic Institute, 94, 1-56.

Lincoln, R.J. (1985b) Deep-sea asellote isopods of the north-east Atlantic: the family Haploniscidae. Journal of Natural History, 19, 655-695. https://doi.org/10.1080/00222938500770411

Meyer-Löbbecke, A., Brandt, A. \& Brix, S. (2014) Diversity and abundance of deep-sea Isopoda along the Southern Polar Front: Results from the SYSTCO I and II expeditions. Deep-Sea Research II, 108, 76-84. https://doi.org/10.1016/j.dsr2.2014.06.006

Malmberg, S.-A. \& Valdimarsson, H. (2003) Hydrographic conditions in Icelandic waters, 1990-1999. ICES Marine Science Symposia, 219, 50-60.

Menzies, R.J. (1962) The isopods of abyssal depths in the Atlantic Ocean. Vema Research Series, 1, 79-206. 
Negoescu, I. \& Svavarsson, J. (1997) Anthurideans (Crustacea, Isopoda) from the North Atlantic and the Arctic Ocean. Sarsia, 82, 159-202.

https://doi.org/10.1080/00364827.1997.10413650

Rothlisberg, P.C. \& Pearcy, W.G. (1977) An epibenthic sampler used to study the ontogeny of vertical migration of Pandalus jordani (Decapoda, Caridea). Fisheries Bulletin, 74, 994-997.

Sneli, J.-A. (1998) A simple benthic sledge for shallow and deep-sea sampling. Sarsia, 83, 69-72. https://doi.org/10.1080/00364827.1998.10413670

Stefánsson, U. (1962) North Icelandic waters. Rit Fiskideildar, 3, 1-269.

Stransky, B. \& Svavarsson, J. (2006) Astacilla boreaphilis sp. nov. (Crustacea: Isopoda: Valvifera) from shallow and deep North Atlantic waters. Zootaxa, 1259, 1-23.

Svavarsson, J. (1988) Bathyal and abyssal Asellota (Crustacea, Isopoda) from the Norwegian, Greenland, and North Polar Seas. Sarsia, 73, 83-106. https://doi.org/10.1080/00364827.1988.10420676

Svavarsson, J., Strömberg, J.-O. \& Brattegard, T. (1993) The deep-sea asellote (Isopoda, Crustacea) fauna of the Northern Seas: species composition, distributional patterns and origin. Journal of Biogeography, 20, 537-555. https://doi.org/10.2307/2845725

Wägele, J.-W. (1983) The homology of antennal articles in Isopoda. Crustaceana, 45 (1), 31-37. https://doi.org/10.1163/156854083X00163

Weisshappel, J.B. (2001) Distribution and diversity of the hyperbenthic amphipod family Calliopiidae in the different seas around the Greenland-Iceland-Faeroe-Ridge. Sarsia, 86, 143-151. https://doi.org/10.1080/00364827.2001.10420469

Wolff, T. (1962) The systematics and biology of bathyal and abyssal Isopoda Asellota. Galathea Report, 6, 1-320. 